\title{
PRACTICAL PHYSIOLOGY POWER LAB. EXPERIMENTS FOR MEDICAL STUDENTS
}

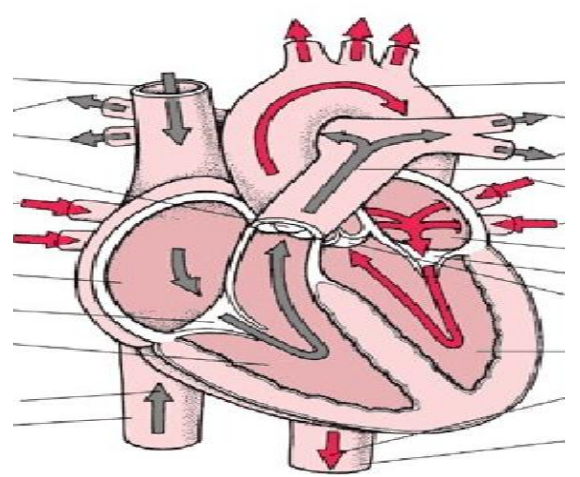

By

Prof. ELSayed A. M. Shokr

PROFESSOR OF PHYSIOLOGY,DEPARTMENT OF PHYSIOLOGY FACULTY OF MEDICINE, HAIL UNIVERSITY

dr.elsayedshokr@yahoo.com

\section{Preface}

With recent advances in the fielded of human physiology, it has become urgent to provide an up to date review in the subject of Power Lab instrument.

This book to help medical student in understanding the human physiology and how to measure the all physiological parameters with the Power Lab instrument. It presents the whole subject in brief comprehensive and up to date form.

We hope this book will be a real help to undergraduate medical students, as well as to postgraduate and candidates of higher degree, in the field of human physiology

Prof. ELSayed A. M. Shokr PROFESSOR OF PHYSIOLOGY DEPARTMENT OF PHYSIOLOGY

FACULTY OF MEDICINE HAIL UNIVERSITY

\section{Council for Innovative Research}

\author{
Peer Review Research Publishing System
}

\section{Journal: Journal of Advances in Chemistry}

Vol. 12 , No. 3

\section{editor@cirjac.com}




\section{Contents}

\begin{tabular}{|c|c|c|}
\hline No. & Title & Pages \\
\hline 1 & Introduction & 4 \\
\hline 2 & Experiment 1 Blood Pressure & 15 \\
\hline 3 & Experiment 2 Breathing & 24 \\
\hline 4 & Experiment 3 ElectroCardioGraph and Heart sound & 39 \\
\hline 5 & Experiment 4 ElectroCardioGraph and peripheral Circulation & 52 \\
\hline 6 & Experiment 5 ElectroEncephaloGraphy (EEG) & 67 \\
\hline 7 & Experiment 6 ElectroMyoGraphy (EMG) & 78 \\
\hline 8 & Experiment 7 Electro- Oculo G raphy (EOG) & 91 \\
\hline 9 & Experiment 8 Muscle & 102 \\
\hline 10 & Experiment 9 Reflexes and Reaction Times & 113 \\
\hline 11 & Experiment 10 Cardiovascular Effects of Exercise & 132 \\
\hline
\end{tabular}

\section{Introduction}

LabTutor is an HTML-based software package, designed specifically for laboratory teaching and used in conjunction with ADInstruments' PowerLab.

LabTutor controls the sampling, digitizing and storage of experimental data, and allows you to display, manipulate and analyze them. The technology derives from equipment such as the Ludwig drum kymograph which recorded data on a revolving drum. This was replaced by paper chart-recorders, such as the Grass polygraph. Then came computer-based data acquisition systems. One of the first of these was MacLab, the predecessor of the ADInstruments PowerLab/LabTutor system, which you are using in this course.

The principle of displaying data in the manner of a paper-chart recorder has not changed. A LabTutor display panel is essentially a window showing a section of a conceptual strip of electronic data chart.

\section{Learning Objectives}

By the end of today's laboratory you will be:

- Familiar with the major hardware and software components of the LabTutor system

- Able to record basic finger-pulse signals in LabTutor

- Able to use some of the annotation and analysis features in the LabTutor software

1890s

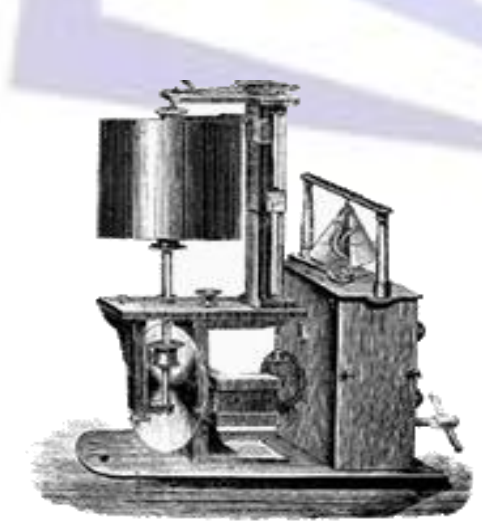

Drum kymograph according to Ludwig 1960s

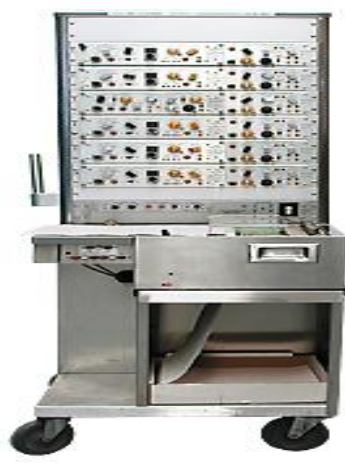

Grass polygraph

\section{Today}
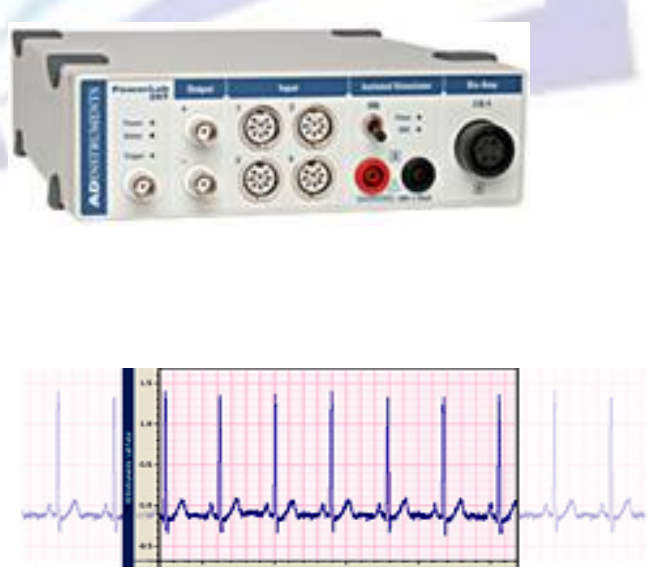

The PowerLab and a LabTutor panel, displaying a section of recorded data 


\section{Data Acquisition}

First, a transducer converts the signal of interest (for example blood pressure or body temperature) into an analog voltage, whose amplitude usually varies over time. This in turn is monitored by the recording hardware, which can modify the signal by amplification and filtering, processes called 'signal conditioning'. The resulting signal is sampled at regular intervals and converted from analog to digital form before transmission to the attached computer, where the sampled data is stored and displayed.

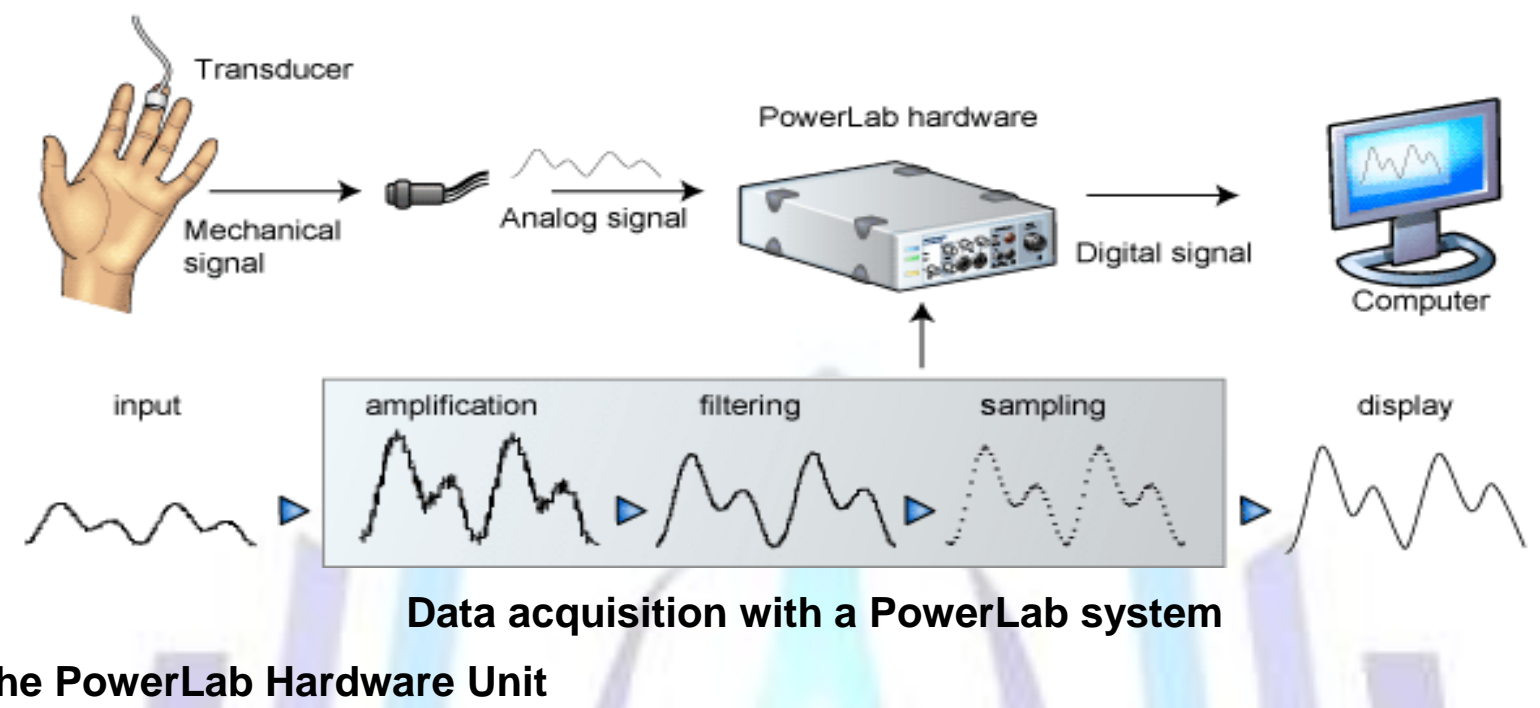

The basic hardware unit is the PowerLab, a multi channel recording instrument for the measurement of electrical signals. Many of the teaching models of PowerLabs include an isolated stimulator for electrical stimulation of nerve and muscle, as well as integrated two channel Bio Amps for optimal recording of biological signals.

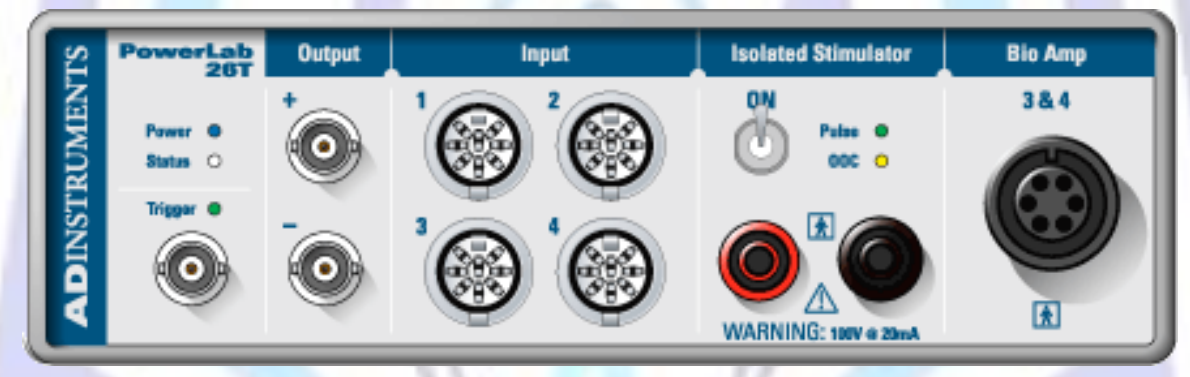

In your experiments, you simply attach appropriate cables to connectors on the front of the PowerLab and measure the signals in LabTutor.

\section{Signal Conditioners}

ADInstruments signal conditioners are software-controlled pre-amplifiers for use with PowerLab data acquisition systems. The two types you are likely to see are Pods and Front-ends. Once connected, the signal conditioners are automatically identified by the PowerLab system, and all settings are stored when the data file is saved on your computer.

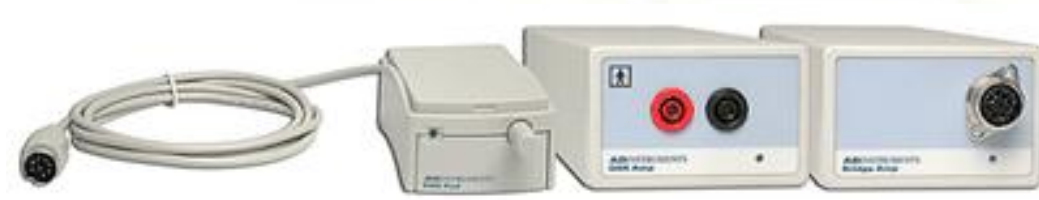

\section{Types of signal conditioners you may use in this course}

Left to right:

Pod used for electro-oculography;

Amplifier for measuring galvanic skin resistance;

Bridge amplifier for connection to force and pressure transducers. 


\section{Signal Transducers}

Virtually any transducer that generates an analog voltage between $\pm 10 \mathrm{~V}$ may be attached to the PowerLab. Either directly or through one of the signal conditioners mentioned above.
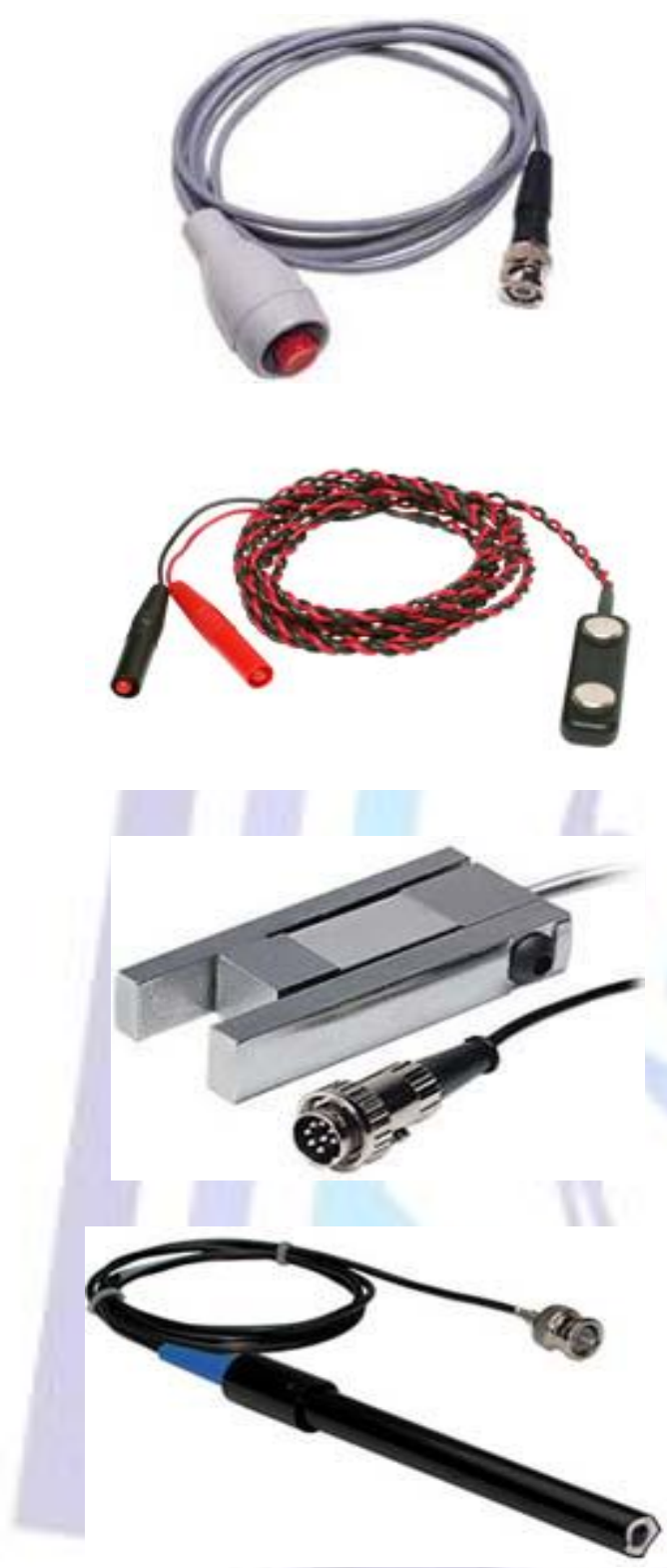

\section{Event markers}

Some transducers may be used to mark an event, like this pushbutton switch.

\section{Stimulation of nerves and muscles}

PowerLab also has the ability to produce an output voltage to induce a response in a nerve or muscle. This stimulating bar electrode connects to the Isolated Stimulator on the PowerLab.

\section{Force or pressure transducers}

Some transducers measure force like this hand dynamometer.

\section{Chemical properties}

Chemical properties may also be measured such as conductivity, dissolved oxygen, and $\mathrm{pH}$.

\section{Connecting Your PowerLab}

Any number of transducers may be connected to the available inputs.

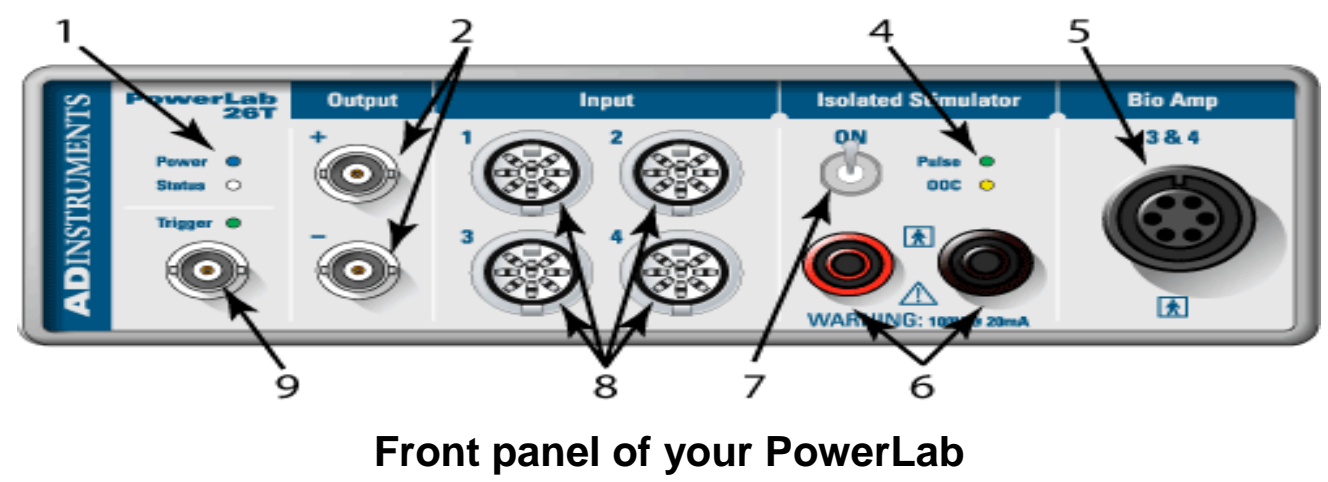


1. Power indicator light: Illuminates when PowerLab is turned on.

2. Analog output connections: provides a voltage output in the range $10 \mathrm{~V}$. NOT safe for direct connection to humans.

3. Analog inputs: Inputs on the PowerLab for connecting transducers and devices with BNC connectors (not present on all models).

4. Isolated Stimulator status light: Indicates that the Isolated Stimulator is working properly (green) or out of compliance (yellow).

5. Dual Bio Amp input: Connects a 5 lead Bio Amp cable to the PowerLab; reads as Inputs 3 and 4. Safe for direct connection to humans.

6. Isolated Stimulator outputs: For connecting stimulating electrodes to the Isolated Stimulator. Safe for direct connection to humans.

7. Isolated Stimulator switch: Turns the Isolated Stimulator on and off.

8. Pod ports: 8-pin connectors for attaching Pods and certain transducers to Inputs. These also supply a DC power to the pods and transducers.

9. Trigger input, may be used to start or stop a recording event (not present on all models).

DO NOT connect a device to the Analog Input and to the Pod port on the same channel.

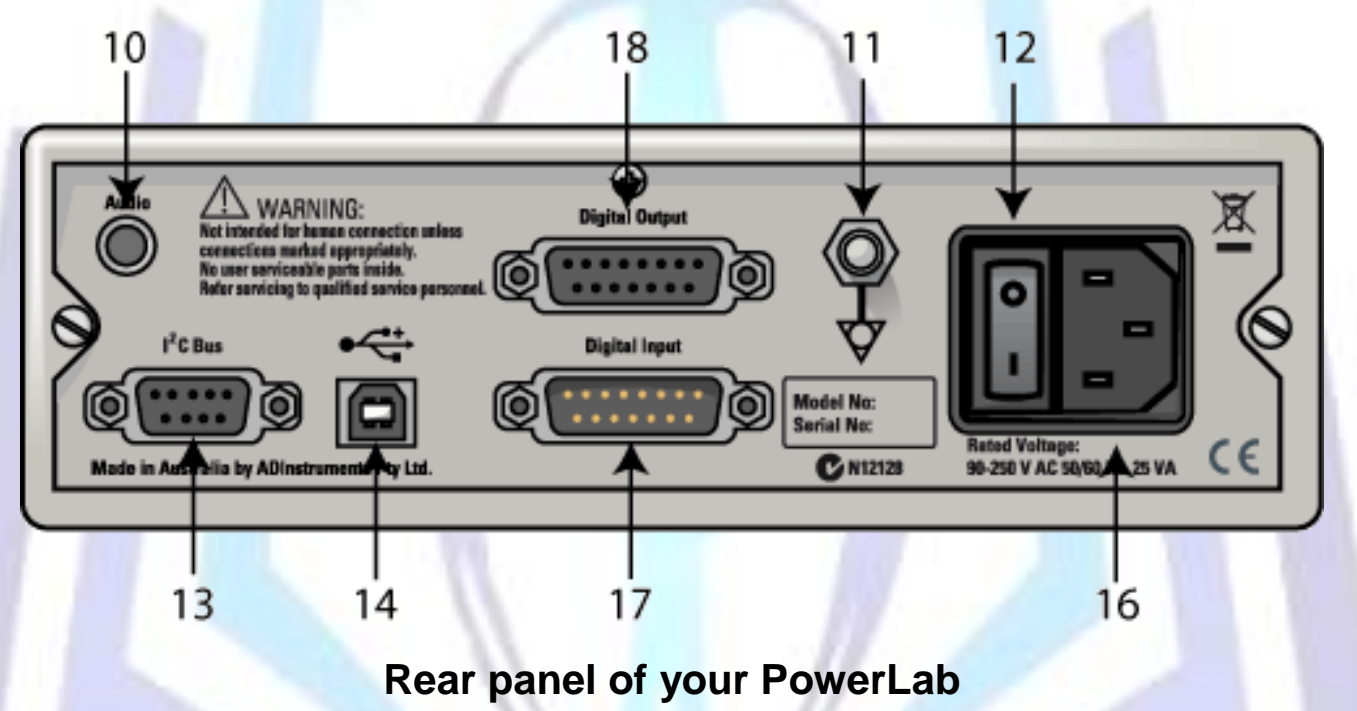

10. Audio output connector: Standard 1/8" (3 mm) phono jack for sound output of recordings from the Bio Amp (not present on all models).

11. Earthing post: Used to ground PowerLab, if grounded power supply is unavailable.

12. Power switch.

13. $I^{2} \mathrm{C}$ connector: Connects PowerLab to special ADInstruments signal conditioners called front-ends (not present on all models).

14. USB connector: for connecting a computer to the PowerLab.

15. Serial Port connector: Connects PowerLab to certain devices (not present on all models).

16. Power cord connector.

17. Digital Input connector (not present on all models).

18. Digital Output connector (not present on all models).

\section{Organization of LabTutor Experiments}

Every LabTutor experiment is organized in basically the same way.

From the experiment index you will find a link to the introductory page of the assigned experiment which may already be pre-loaded onto your computer.

Every experiment begins with an introductory page. On this page there is a brief introduction and a list of learning objectives. The subsequent exercises allow you to accomplish the specified learning objectives. 
Each exercise includes highlighted text with links to pop-up windows containing additional information, helpful tips, and useful references to LabTutor features.

Each exercise page contains a LabTutor panel in which data is recorded.

After each Exercise page, there is an Analysis page. Data that you recorded during the exercise is available here for you to make measurements and complete any tables or graphs that are required.

At the end of the experiment is the Report section. Any recordings that are required for your report are reproduced here, along with tables or graphs that you have completed. This section also contains questions that you can answer by typing into the spaces provided. Your instructor will advise you how to submit your completed lab report.

All pages include a link in the footer to the Background material. This provides some general information regarding the science behind the experiment. It may have been given to you by your instructor prior to your laboratory.

\section{Procedure}

1. Make sure the PowerLab is connected and turned on.

2. Place the pressure pad of the finger pulse transducer against the distal segment (the tip) of the middle finger or thumb of either hand. Use the Velcro strap to attach it firmly - neither loose nor tight.

If the strap is too loose, the signal will be weak, intermittent, or noisy. If the strap is too tight, this will reduce blood flow to the finger, thus weakening the signal, and may also cause discomfort. You may need to adjust the strap in the next stage of the exercise.

3. Connect the plug from the finger pulse transducer's cable to the socket for Input 1.

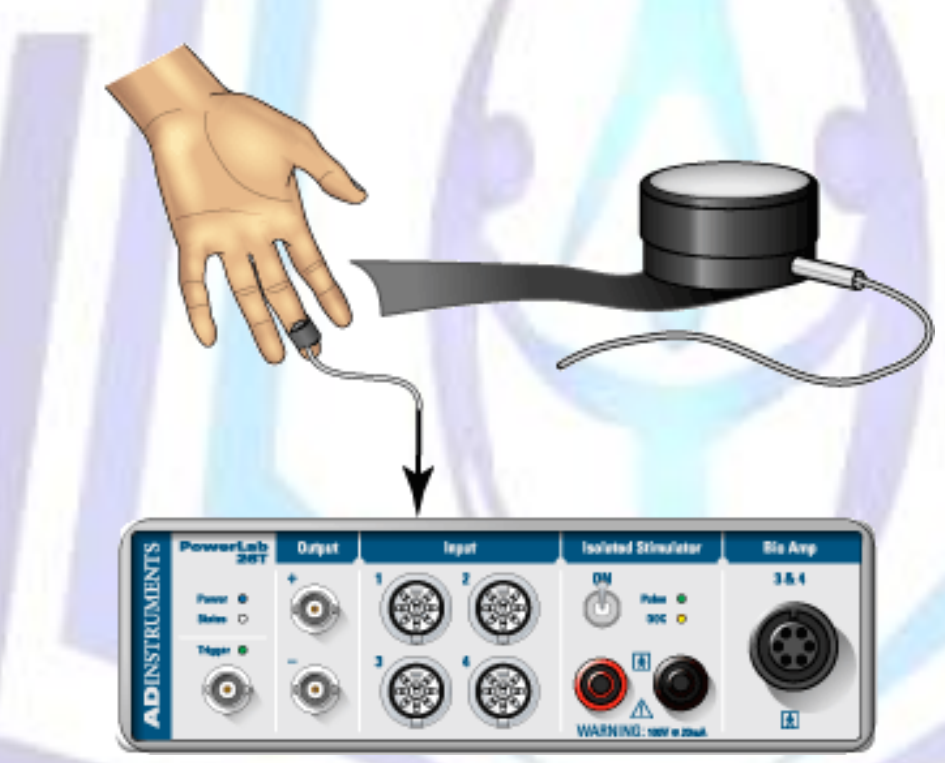

\section{Recording Data}

1. Click the Start button in the top right-hand corner of the LabTutor panel to begin recording your finger pulse data.

2. Click the Autoscale button at the top of the LabTutor panel. The data will now be scaled to occupy most of the height of the channel. You will use the Autoscale button frequently to optimize the display of your data.

3. After about 20 seconds, click Stop. The data that you have recorded is automatically saved when you stop recording.

4. Your record should now resemble this.

\section{Scrolling}

The scroll bar allows you to move back and forward through your file. You can think of your recording as a large strip of paper scrolling past the LabTutor panel.

The scroll bar is primarily an analysis tool, allowing you to review and locate data of interest anywhere within a file.

During recording you can also review your data, without stopping recording, by initiating Scroll Review mode. Scroll Review mode is engaged by either dragging the scroll bar thumb to the left or clicking anywhere in the incoming data. To return to normal scrolling mode click the Move to End of Data button. 
Generally, while recording, you will be more interested in examining the incoming signal than reviewing earlier recorded data.

\section{Horizontal Compression Buttons}

With the Horizontal Compression buttons, located at the bottom left of a LabTutor panel, you can compress or expand the Time axis to see more or less of the recorded data.

Click a few times on the Compression buttons to see what this does to the display of your data. The extent of compression is displayed as a ratio on the Ratio button, which is located between the Compression buttons.

Click the Ratio button: a pop-up menu appears; from this you can choose the compression directly.

\section{Vertical Scaling Buttons}

The Vertical Scaling buttons are on the left side of each channel's Amplitude axis. These buttons allow you to compress or expand the visible part of the vertical scale in each channel independently.

If you move the pointer over the scale part of the Amplitude axis, small arrows appear beside the pointer. You can either stretch or move the scale by dragging the scale numbers or the scale between them. The small arrows beside the pointer indicate what will happen.

\section{Autoscale and Default Scale Buttons}

The Autoscale button adjusts the height of the scaled data to display the minimum and maximum data. Autoscale adjusts the display based on the data visible on screen.

The Default Scale button resets the vertical scale and horizontal compression to their original settings. This can be useful if, during the course of your experiment, you lose focus on your data because you have manipulated the vertical scale beyond the signal limits.

\section{Annotating a Record}

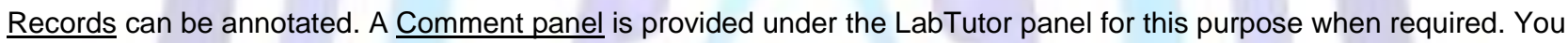
can add comments either while you are recording, or after you have finished.

\section{Procedure}

1. Click Start in the LabTutor panel to start recording.

2. Click in the Comment panel text-box, and type in some text.

3. Click the Add button. The text disappears and a vertical dotted line appears in the LabTutor panel.

4. Repeat steps 2 and 3 to add a second comment.

5. Click Stop.

After you have finished recording, you will see numbered Comment boxes in the LabTutor panel. Now try these:

- $\quad$ Click a Comment box: the text you typed appears in the pop-up panel. Edit the comment by typing new text into the comment panel and clicking the Edit button.

- Add comments after recording has finished. Click in the LabTutor panel at the place where you wish to insert the comment and then proceed as outlined in steps 2 and 3 above.

\section{Making Measurements}

You can make measurements and add the relevant numbers into a Table.

1. Place the cursor over the data at the desired point and click to place the selected data in the Value panel.

2. To insert a value into a table, drag it from the Value panel into the appropriate cell of the table.

3. You can also type information, including values, into any cell in the Table.

4. Complete the Table using the data you have already recorded, by clicking a peak of the pulse recording and transferring the time and amplitude values into the first row of the table. Repeat for the next three peaks of the pulse.

\section{The Marker}

When the Marker is in use, values and times displayed in the Value panel are relative to the Marker position. When not in use, the Marker resides in a dock at the bottom left of the LabTutor panel; from here it can be dragged and dropped on to any part of the data. To return the Marker to its dock, simply click in the dock area. 
1. Drag the Marker from its home and drop it somewhere on the trace. The Marker does not have to be placed exactly on the waveform. When released, the Marker will drop and attach itself to the waveform.

Values placed in a Value panel will now be prefaced by a $\Delta$ (delta) symbol, to indicate that the measurement is relative to the Marker's position.

2. Complete the Table using the Marker. Place the Marker on a peak of the pulse recording. Click on the following trough to add the values for time and amplitude to their respective Value panels. Drag these values into the table and repeat for the next three peaks of the pulse (see example).

3. Remove the Marker from the waveform by clicking in the Marker dock.

\section{Calculations}

LabTutor can be configured to calculate variables based on the raw signal input from other channels. These can be displayed in real time on channels you are not using for data acquisition.

\section{Procedure}

1. Click Start in the LabTutor panel to start recording.

2. Click in the Comment panel text-box, and type in the volunteer's name.

3. Observe the following traces as they appear on the screen:

- Channel 1 is the finger pulse

- Channel 2 displays the interval between peaks of the pressure waves.

- Channel 3 displays the calculated heart rate in Beats Per Minute.

\section{Click $\underline{\text { Stop }}$}

When more than one channel is displayed:

- You can change the channel heights by dragging the channel separators up or down.

- You can restore the original channel heights by double-clicking on any channel separator.

\section{Deleting Data}

LabTutor automatically saves your data. Occasionally you may want to discard a segment of your trace or delete some noisy data.

This action cannot be undone!

\section{Procedure}

1. Scroll through the data you just recorded and find a section that appears excessively noisy. Click the Auto Scale or Default Scale button, as necessary.

2. Click and drag over the portion of the trace you want to remove.

You will notice that LabTutor automatically selects the corresponding data from all channels displayed on the screen. You cannot delete data from a single channel; this ensures that the time record always corresponds to the voltage value recorded.

3. Click the Clear Selection button.

This section contains your results and analysis, together with questions about them. It can be printed and submitted to your instructor if required. It shows how LabTutor panels and tables from the exercises are automatically duplicated in the Report.

\section{PowerLab Hardware and Transducers}
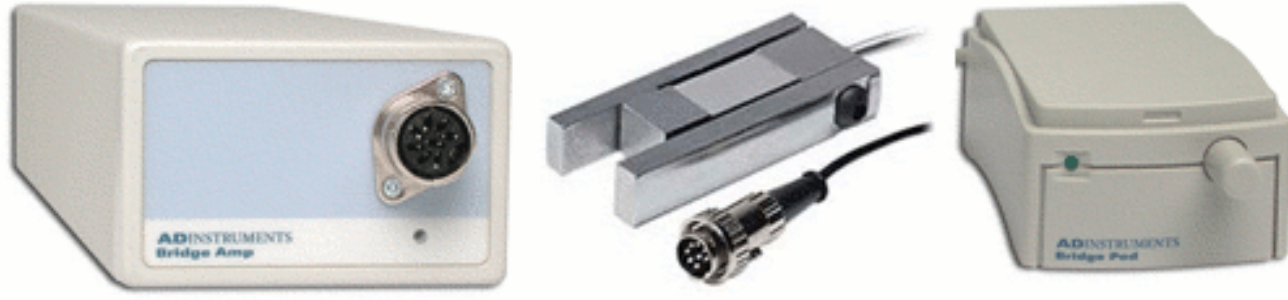

1. In your own words, briefly describe the function of the PowerLab components shown above. 


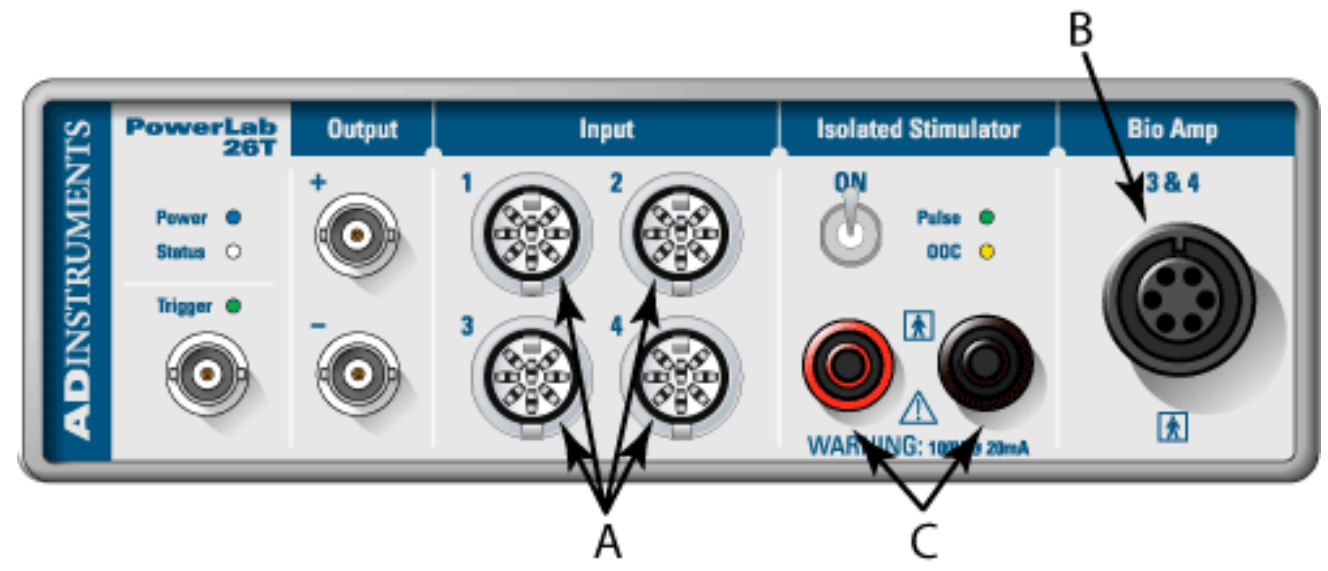

2. In your own words, describe the function of the labeled parts of the PowerLab shown above.

\section{Recording of a Finger Pulse}

1. Today you used a finger pulse transducer to collect some physiological data and make some recordings. In your own words, being as specific as you can, describe what is actually being recorded by the PowerLab and displayed in LabTutor.

2. Name two physiological variables, other than finger pulse, that you could measure with PowerLab and LabTutor.

3. Were all of your measurements identical for every member of your group? Would you expect them to be identical?

\section{Deleting Data and Channel Calculations}

1. Can you think of some other parameters that LabTutor could calculate based on your original pulse trace?

2. Why is it important that the data in all channels be deleted simultaneously?

\section{Introduction}

\section{Experiment 1}

\section{Blood Pressure}

In this laboratory, you will become familiar with auscultation (listening to the sounds of the body) and the measurement of blood pressure. The exercises involve measuring your blood pressure using a stethoscope, blood pressure cuff and sphygmomanometer. You will also assess changes in peripheral circulation and the effects of cuff location.

The modern era of blood pressure measurement started with the introduction of the mercury sphygmomanometer by Scipione Riva-Rocci (1863-1937) in 1896.

\section{Learning Objectives}

By the end of today's laboratory you will be able to:

- Use a sphygmomanometer and stethoscope to measure human arterial blood pressure

- Determine systolic blood pressure using a sphygmomanometer and detection of a peripheral pulse

- Demonstrate how measurement position affects the magnitude of the arterial blood pressure

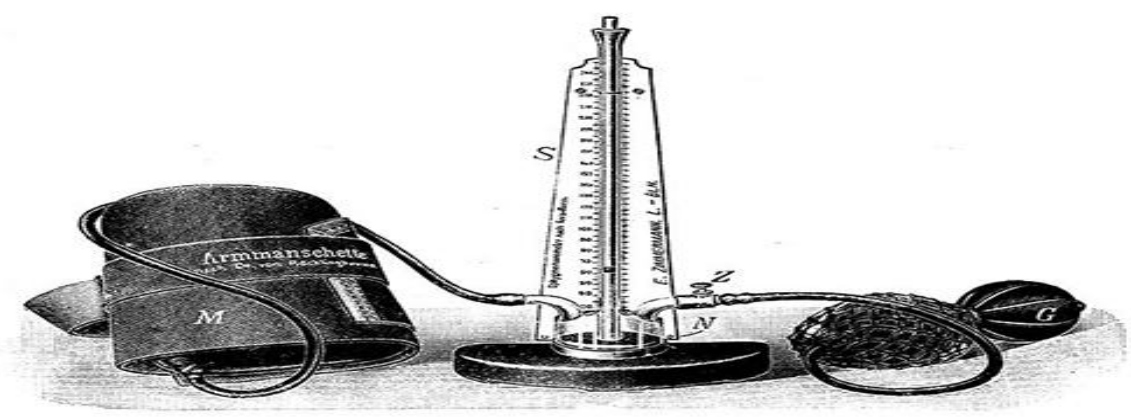

Riva-Rocci style sphygmomanometer based on the original 1896 design. 


\section{Procedure}

To perform this experiment correctly, you must be familiar with the use of the stethoscope and sphygmomanometer.

\section{Caution}

The sphygmomanometer conveniently combines a cuff and bulb with a pressure transducer.

1. Plug the pressure transducer into Input 1 on the PowerLab.

2. Plug the Cardio Microphone into Input 2.

3. Wrap the sphygmomanometer cuff around the upper arm just above the elbow as shown.

4. Click $\underline{\text { Start. }}$

5. Practice inflating the cuff to approximately $180 \mathrm{mmHg}$ and slowly reducing the pressure (1-2 $\mathrm{mmHg}$ per second) until you are confident that you can use the sphygmomanometer correctly.

6. Click Stop.

\section{Exercise 1}

In this exercise you will measure blood pressure in the traditional way, using a stethoscope to listen for Korotkoff sounds.

\section{Procedure}

1. Inflate the cuff until the pressure reaches approximately $180 \mathrm{mmHg}$.

2. Slowly reduce the pressure in the cuff (approximately 1 to $2 \mathrm{mmHg}$ per second) while listening through the stethoscope for Korotkoff sounds.

3. The systolic pressure is the pressure at which sharp, tapping sounds are first heard.

4. Continue slowly reducing cuff pressure (at 1 to $2 \mathrm{mmHg}$ per second). The diastolic pressure is defined as the pressure at which the sounds disappear.

5. Completely deflate the cuff once diastolic pressure is determined. Do not leave the cuff partially inflated or leave it inflated for a long time.

6. For each subject, record four measurements of the blood pressure. Allow one to two minutes between measurements for recovery.

7. Repeat the procedure using other students until you feel confident in measuring blood pressure.

\section{Exercise 1: Auscultation}

\section{Study Question}

1. What is your systolic blood pressure measurement?

2 . 2 - What is your diastolic blood pressure measurement?

\section{Exercise 2}

In this exercise you will use the Cardio Microphone to record arterial sound while recording blood pressure.

\section{Procedure:}

1. Leave the blood pressure cuff in place around the upper portion of the student's arm (either arm), between the elbow and the shoulder.

2. Place the Cardio Microphone over the brachial artery and under the blood pressure cuff so that it is held in position by the cuff.

3. Click Start.

4. Inflate the cuff until the pressure reaches approximately $180 \mathrm{mmHg}$.

5. Slowly reduce the pressure in the cuff (approximately 1 to $2 \mathrm{mmHg}$ per second). Deflate the cuff completely once the pressure has gone below $50 \mathrm{mmHg}$.

6. Click Stop.

7. Repeat the procedure using other students. Remember to add a comment with the subject's name for later identification. Allow one to two minutes between procedures for recovery. 


\section{Analysis}

1. Examine your recording. The Cardio Microphone channel displays the Korotkoff sounds as spikes. These spikes can be used to determine systolic and diastolic pressure.

2. Place the Waveform Cursor on the the first spike following the reduction in cuff pressure. This represents the systolic pressure.

3. Click on this point to enter the pressure in the value panel and add the comment "systolic pressure" to the data.

4. Drag the number from the value panel into the appropriate column of the table.

5. Place the Waveform Cursor on the the last spike in the series. This represents the diastolic pressure.

6. Click on this point to enter the pressure in the value panel and add the comment "diastolic pressure" to the data. In some subjects it is not possible to determine diastolic pressure using this technique.

7. Repeat these procedures for all subjects in your group

\section{Exercise 2: Cardio Microphone}

\section{Study Questions}

1. What is your heart sound write what are you listen?

\section{Exercise 3}

You will observe the changes in finger pulse while measuring blood pressure, and see if pulse measurement could replace the use of the stethoscope.

\section{Procedure}

1. Remove the Cardio Microphone plug from Input 2 on the PowerLab.

2. Connect the finger pulse transducer to Input 2.

3. Place the pressure pad of the finger pulse transducer against the distal segment (the tip) of the middle finger of your hand (on the same arm as the blood pressure cuff). Use the Velcro strap to attach it firmly - neither loose nor tight.

4. Relax, put your hands in your lap and sit as still as possible to minimize any movement artifact.

5. Click Start, the recorded pulse should look something like this.

6. Add a comment with the subject's name.

7. Inflate the cuff until the pressure is just above $180 \mathrm{mmHg}$. Note that the pulse signal disappears.

8. Slowly deflate the cuff at a rate of 1 to $2 \mathrm{mmHg}$ per second.

9. Once the pressure has reached $50 \mathrm{mmHg}$, completely deflate the blood pressure cuff.

10. Click Stop.

\section{Analysis}

1. Examine your recording. Place the Waveform Cursor on the the first finger pulse seen as the cuff pressure was falling. This represents the return of bloodflow to the forearm.

2. Click on this point to enter the pressure in the Value panel and add the comment "systolic pressure" to the data.

3. Drag the number from the value panel into the appropriate column of the table.

\section{Exercise 3: Blood pressure and pulse}

\section{Study Questions}

1. Comment on how the systolic pressure determined by auscultation and by pulse detection compare for each of the subjects in your group.

2. From your results, do you think that pulse measurement can replace the stethoscope in determining diastolic pressure? 


\section{Exercise 4}

This exercise is a variation on Exercise 3, with measurements taken from a different site on the arm and with the arm in different positions.

\section{Procedure}

1. Wrap the cuff around the forearm, immediately above the wrist, of the same hand which has the finger pulse transducer attached.

2. Ensure the subject's elbow is flexed at 90 degrees, with the wrist resting on a chair arm or desk.

3. Type "arm resting, 90 " into the comment panel.

4. Click Start.

5. Inflate the cuff to $180 \mathrm{mmHg}$.

6. Press Add to enter the initial comment for this exercise.

7. Slowly deflate the cuff at a rate of 1 to $2 \mathrm{mmHg}$ per second.

8. Once the pressure has reached $50 \mathrm{mmHg}$, completely deflate the blood pressure cuff.

9. Click Stop.

10. Repeat steps 3 to 9, entering an appropriate descriptive comment each time, with the arm in the following positions:
○ Hanging down loosely by the side
- Held straight above the head.

\section{Analysis}

Determine the systolic blood pressure from the pressure cuff and finger pulse data.

1. Examine the finger pulse data. Place the Waveform Cursor on the the first pulse following the reduction in cuff pressure. This represents the systolic pressure.

2. Click on this point to enter the pressure in the Value panel and add the comment "systolic pressure" to the data.

3. Drag the number from the value panel into the appropriate column of the table.

4. Repeat steps 1-3 for each of the protocols in the exercise.

\section{Exercise 4: Hydrostatic effects}

\section{Study Questions}

1. Explain the differences in pressure with the arm in different positions.

2. Given the variation in pressure with height, in clinical medicine human blood pressure is normally referenced to heart level. Does this usually affect the pressure recorded from the upper arm?

\section{Experiment 2}

\section{Breathing}

\section{Introduction}

In this laboratory, you will record breathing movements with a respiratory belt transducer fastened around the abdomen. You will investigate various aspects of breathing, including the ability to hold the breath, hyperventilation, rebreathing, and the relation between breathing and heart rate.

\section{Learning Objectives}

By the end of today's laboratory you will be able to describe and explain the:

- Effects on breathing of voluntary hyperventilation

- Effects on the respiratory pattern of rebreathing expired gas

- $\quad$ Relationship between breathing and heart rate 


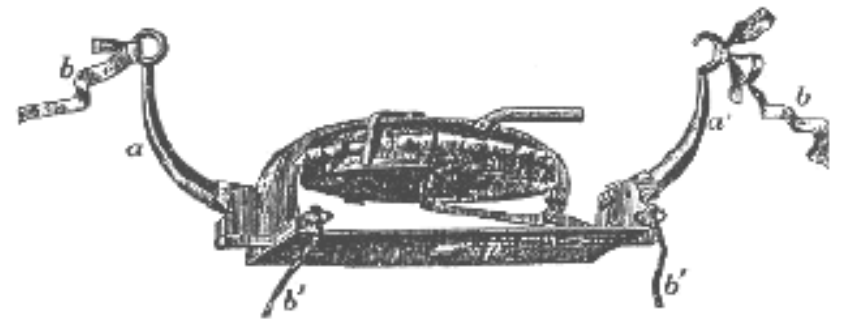

This device was developed by the French scientist Étienne- Jules Marey (1830-1904) to indicate movements of the chest due to breathing. It is mounted on a spring plate which returns to its original position when the breath is expired.

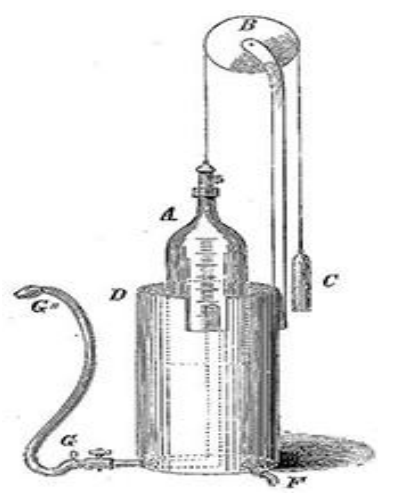

An example of an early spirometer: Hutchinsons spirometer.

\section{Procedure}

1. Fasten the respiratory belt around the abdomen of a volunteer, as shown. The transducer should be:
- At the front of the body, level with the navel.
- Tightened sufficiently that it remains under tension even when the subject fully exhales.

The respiratory belt transducer can be used over clothing, and it doesn't matter whether the volunteer is sitting or standing, so long as they are comfortable (this is quite a long exercise). Because breathing patterns differ, you may need to reposition the transducer over the chest rather than the abdomen to get the best signal.

2. Connect the plug on the respiratory belt transducer cable to Input 1 on the front of the PowerLab.

It is important when recording normal respiration that the volunteer is facing away from the computer screen and is not consciously controlling breathing. The volunteer may have to stare out a window or read a book to avoid conscious control of respiration.

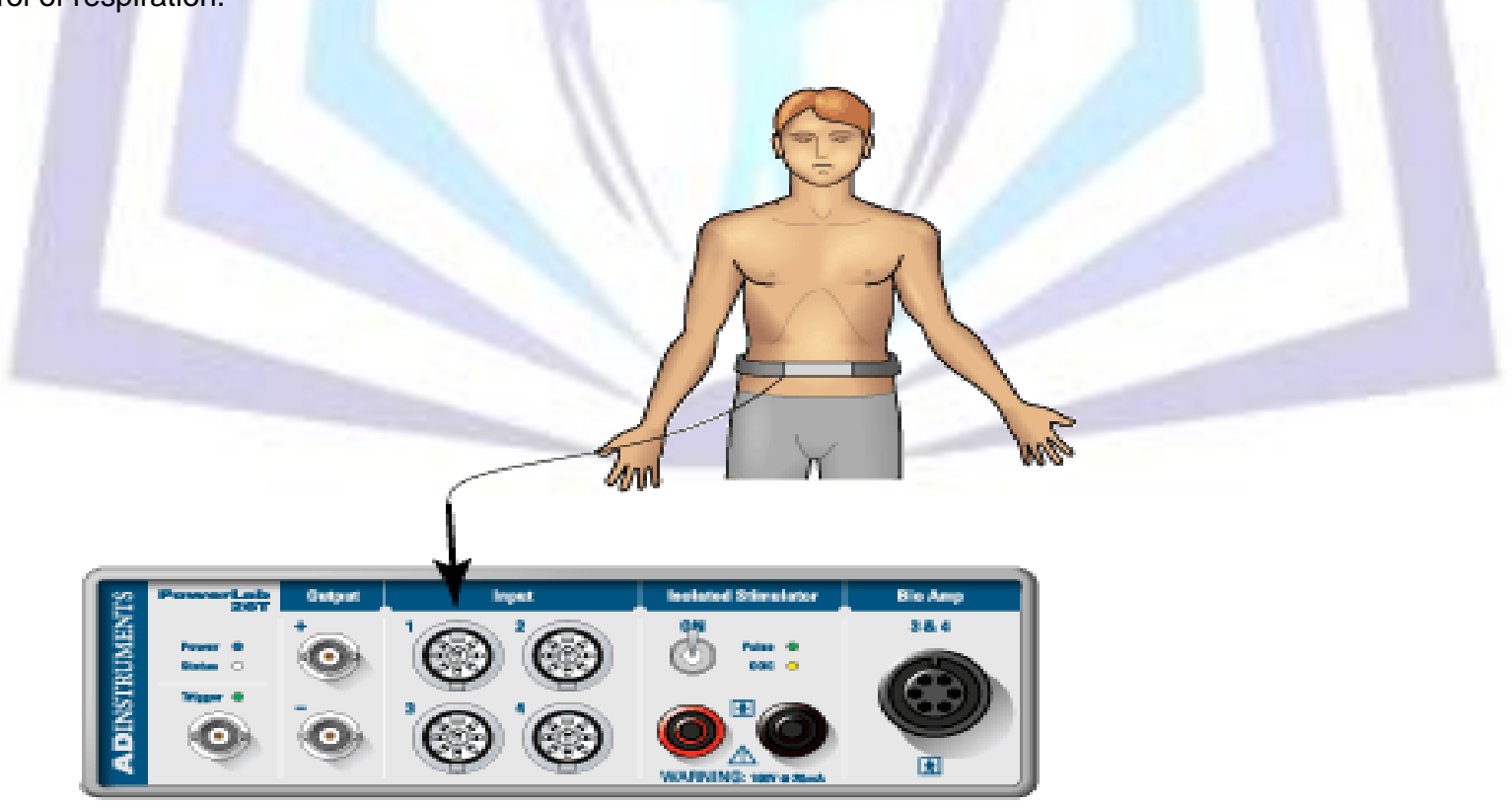

\section{Exercise 1}

In this exercise, you will record normal and rapid breathing, and examine the effects of holding your breath after inhaling and after exhaling. 


\section{Procedure}

1. Click Start.

2. Ask the volunteer to breathe rapidly for a few seconds, and then to breathe slowly. Examine the Breath Rate channel, there should be obvious changes in rate.

3. Enter a comment: 'Baseline 1' in the slow breathing region of the data.

4. Click Add.

5. Record 2-3 minutes of normal, quiet breathing and observe the trace.

6. Enter a comment: 'inhale, hold'.

7. Click Add, and immediately ask the volunteer to take a deep breath and hold it in for as long as possible.

8. Enter a comment: 'breathe'.

9. When the volunteer begins to breathe again, click Add .

10. Wait until a normal (baseline) breathing pattern resumes; then let the volunteer rest and breathe normally for another 2-3 minutes.

11. Enter a comment: 'exhale, hold'.

12. Click Add, and immediately ask the volunteer to breathe out fully and hold the breath for as long as possible.

13. Enter a comment: 'breathe'.

14. When the volunteer begins breathing click Add.

15. Continue recording until a normal (baseline) pattern resumes.

16. Click Stop.

The volunteer can now relax and breathe normally.

\section{Analysis}

1. In the Breathing channel, place the Marker on the large peak following the comment 'inhale, hold'.

2. Move the Waveform Cursor to the start of the first breath afterwards, which should be preceded by the comment 'breathe'.

3. Click to place the selected data in the Value panel and drag it from the Value panel into the appropriate cell of the table.

4. Drag the Marker to the large (negative) peak right after the comment 'exhale, hold'.

5. Move the Waveform Cursor to the start of the first breath afterwards, which should also be preceded by the comment 'breathe'.

6. Click to place the selected data in the Value panel and drag it from the Value panel into the appropriate cell of the table.

\section{Exercise 1: Normal respiration}

\section{Study Questions}

1. Describe the normal respiratory movements.

2. what is the value of top of the curve?

3. what is the value of bottom of the curve?

\section{Exercise 2}

In this exercise, you will record the effect of voluntary hyperventilation on breath-holding and the recovery of normal breathing rhythm.

\section{Safety considerations}

\section{Procedure}

1. Click Start.

2. Enter a comment 'baseline', click Add and ask the volunteer to maintain normal respiration for 2-3 minutes. 
3. Enter a comment: 'inhale, hold'.

4. Click Add, and immediately ask the volunteer to take a deep breath and hold it in for as long as possible.

5. Enter a comment: 'breathe'.

6. When the volunteer begins to breathe again, click Add .

7. Record the subject's normal respiration for 2-3 minutes. During this time, enter a comment: 'hyperventilate'.

8. Click Add and immediately ask the volunteer to hyperventilate by breathing as quickly and as deeply as possible for 30 seconds.

9. Enter a comment: 'breathe'.

10. After the 30 seconds of hyperventilation click Add, then immediately tell the volunteer to begin breathing normally again.

11. Wait until a normal breathing pattern resumes; then let the volunteer rest and breathe normally for another 2-3 minutes.

12. Enter a comment: 'hyperventilate'.

13. Click Add, then immediately ask the volunteer to hyperventilate again by breathing as quickly and as deeply as possible for 30 seconds.

14. Enter a comment: 'inhale, hold'.

15. After the 30 seconds of hyperventilation click Add, and Immediately ask the volunteer to take a deep breath and hold it in for as long as possible.

16. Enter a comment: 'breathe'.

17. When the volunteer begins breathing click Add.

18. Click Stop.

The volunteer can now relax and breathe normally

\section{Analysis}

1. In the Breath Rate channel, select an area representing your normal breathing rate prior to the 'inhale, hold' comment. This will give you the average breathing rate for that period of time.

2. Drag the the Breath Rate data from the Value Panel into the appropriate cell of the table.

3. In the Breathing channel, select the period of time for which the subject held their breath (from the 'inhale, hold' comment to the 'breathe' comment), and drag this Breathing Selection Duration data from the Value Panel to the appropriate cell in the table.

4. Repeat steps 1-3 for the hyperventilation period and the subsequent breath-hold.

\section{Exercise 2: Hyperventilation}

\section{Study Questions}

\section{Define hyperventilation.}

2. Measure the difference between hyperventilation and normal ventilation

\section{Exercise 3}

In this exercise, you will observe the effect of rebreathing exhaled gases. You will need to obtain a medium-sized paper bag. When re-breathing, the volunteer should place this so that it covers the nose and mouth and forms a tight seal.

\section{Procedure}

1. Click Start. Enter a comment: 'baseline', and click Add.

2. Record the baseline for 2-3 minutes.

3. Enter a comment: 'rebreathing'.

4. Click Add and immediately ask the volunteer to place the paper bag over the nose and mouth, and rebreathe the air in the bag.

5. Enter a comment: 'breathe'. 
6. After 60 seconds of rebreathing, click Add; then immediately ask the volunteer to remove the paper bag from the nose and mouth.

7. Continue recording for 60 seconds.

8. Click Stop.

\section{Analysis}

Rebreathing from a closed bag results in arterial hypercapnia (raised partial pressure of carbon dioxide), which stimulates respiration. How was this evident in this exercise? (That is, did the depth or rate or both increase during rebreathing compared to normal breathing?)

\section{Procedure}

1. Leave the respiratory belt fastened around the abdomen of the volunteer.

2. Connect the finger pulse transducer to Input 2 on the PowerLab.

3. Place the pressure pad of the finger pulse transducer against the tip of the middle finger of either hand of the volunteer. Use the Velcro strap to attach it firmly - neither loose nor tight.

4. Ensure that the person sits quietly with his or her hands resting in their lap, or on a bench, to minimize transducer movements.

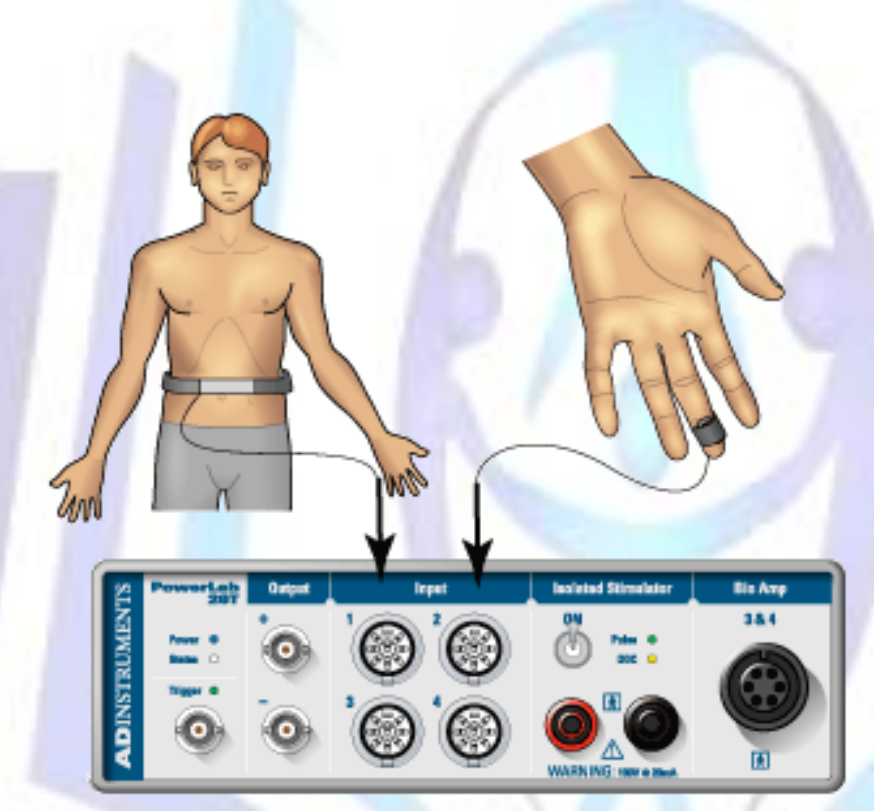

\section{Exercise 3: The effect of rebreathing}

\section{Study Questions}

1. Describe the effects of rebreathing that you observed.

2. 2 - Measure the top and the bottom of the curve of breathing.

\section{Exercise 4}

In this exercise, you will record and examine the effect of breath-holding on heart rate.

\section{Procedure}

1. Click Start.

2. Record a baseline heart rate and breathing pattern for two minutes. (Variation in the heart rate is most evident with slow, deep breathing.)

3. After recording the baseline signals, enter a comment: 'inhale, hold'.

4. Click Add, and immediately ask the volunteer to take a deep breath and hold it in for as long as possible.

5. While the volunteer is not breathing, enter a comment: 'breathe'.

6. When the volunteer begins breathing, click Add. 
7. Click Stop.

The volunteer can now relax and breathe normally.

\section{Analysis}

Heart rate variations within the breathing cycle should be seen best at a timescale compression of 20:1.

\section{Exercise 4: Breathing and heart rate}

\section{Study Questions}

1. In what way does the heart rate change during the breathing cycle?

2. What happened to the heart rate trace while the breath was held

\section{Experiment 3}

\section{ElectroCardioGraph}

and

\section{Heart sound}

\section{Introduction}

The beating of the heart is associated with electrical activity and sound. The pattern of electrical activity recorded at the body surface is called the electrocardiogram or ECG. The aim of this laboratory is for you to record and analyze an ECG from a volunteer, and to examine the relationship between the ECG and the characteristic sounds of the heart.

\section{Learning Objectives}

By the end of today's laboratory you will:

- Know where to place electrodes to record the standard limb lead ECG (leads I, II and III )

- Be able to identify the major components of the ECG (P wave, QRS complex, T wave) in these leads

- Be able to relate the electrical activity in the heart to these major components

- Be able to provide estimates of the timings of the components of the ECG and their magnitudes

- Know how to calculate heart rate from the ECG

- Explain the time relationships between the electrical activity of the heart (as recorded in the ECG) and the mechanical activity of the heart (as judged from the heart sounds)

Instruments for recording the activity of the heart. 

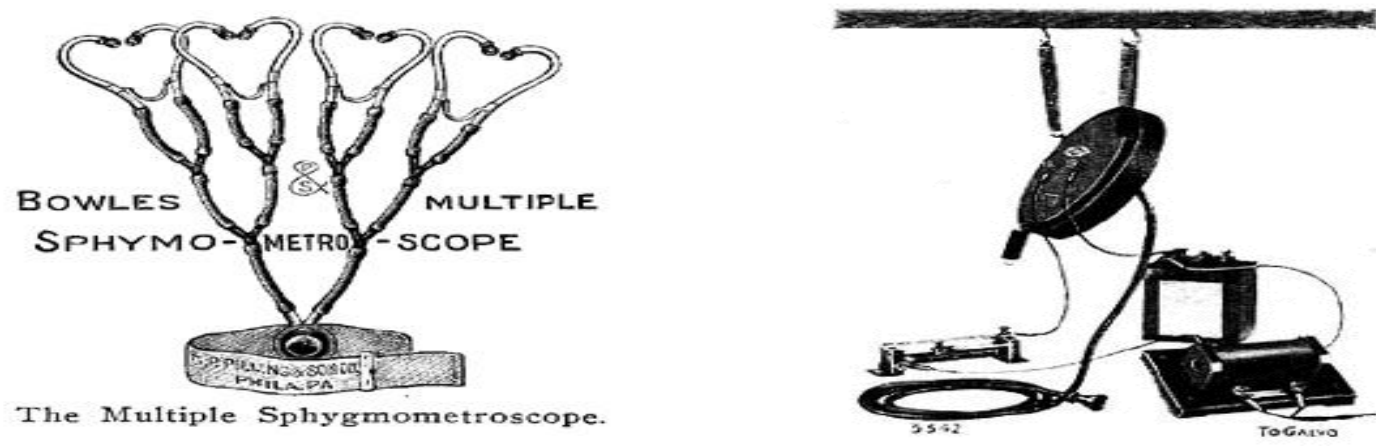

A teaching instrument

An early (1920s) cardiac microphone

\section{Procedure}

1. Make sure the PowerLab is connected and turned on.

2. Connect the push-button switch to Input 1 on the PowerLab.

3. Remove any watches and/or jewelry from your wrists and ankles.

4. Connect the electrode lead wires to Earth, $\mathrm{CH} 1 \mathrm{NEG}$, and POS on the Bio Amp cable.

5. Plug the Bio Amp cable into the Bio Amp input.

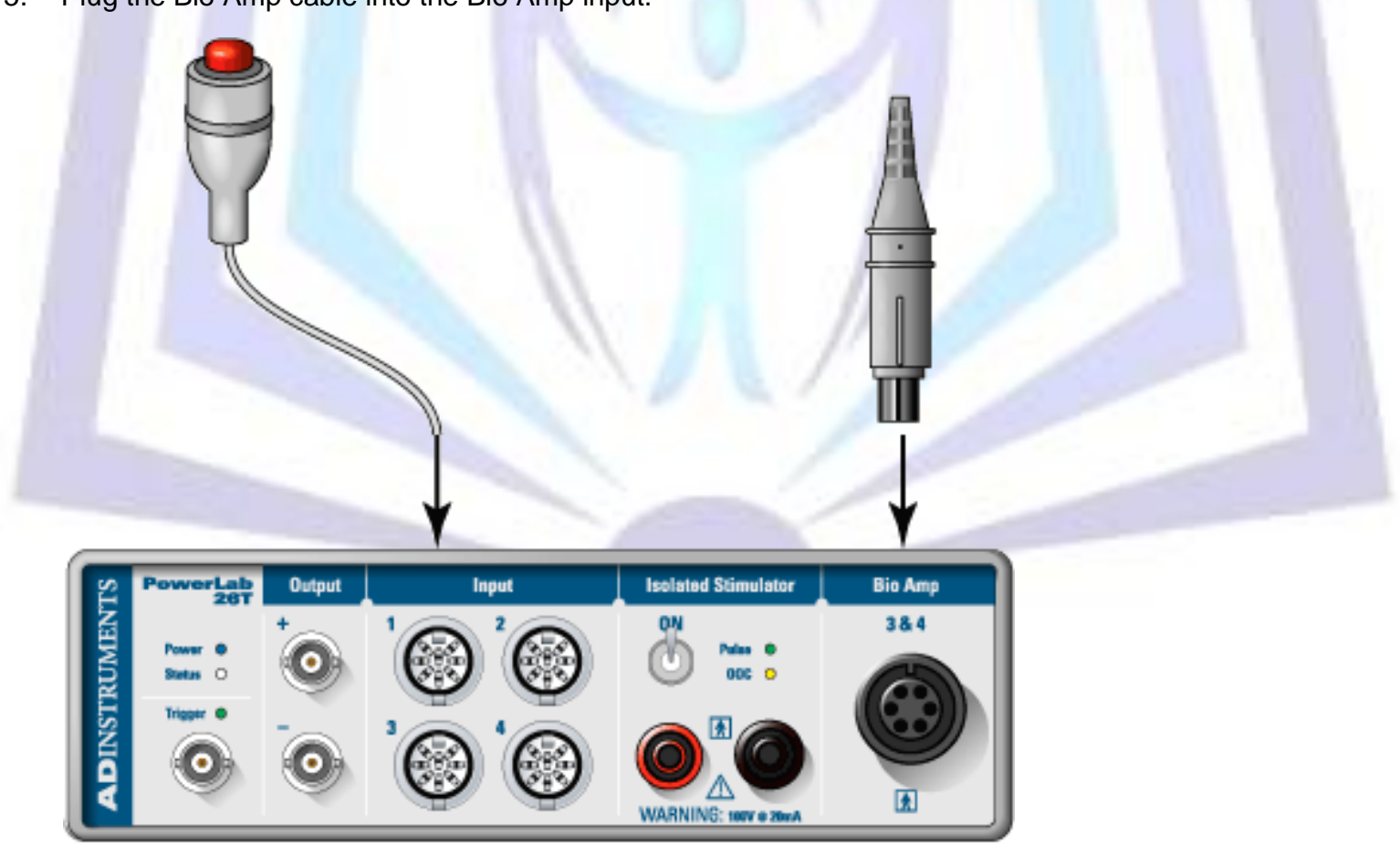

\section{Standard Connection}

Attach the positive electrode to the left wrist, the negative to the right wrist, and the ground to the right leg.

1. Using a pen, mark each point where electrodes will be placed. Clean the skin with alcohol swabs and lightly abrade the area with abrasive gel or a pad. This reduces the electrical resistance of the outer layer of skin and ensures good electrical contact. 
2. If you are using the Reusable Clamp Electrodes, apply a small amount of electrode cream to the electrodes before attaching. Electrode cream is not necessary if you are using disposable electrodes which have electrode gel on them already.

3. If, after looking at the signal during the first exercise, you find that this does not produce a good signal, try the alternative method.

\section{Exercise 1}

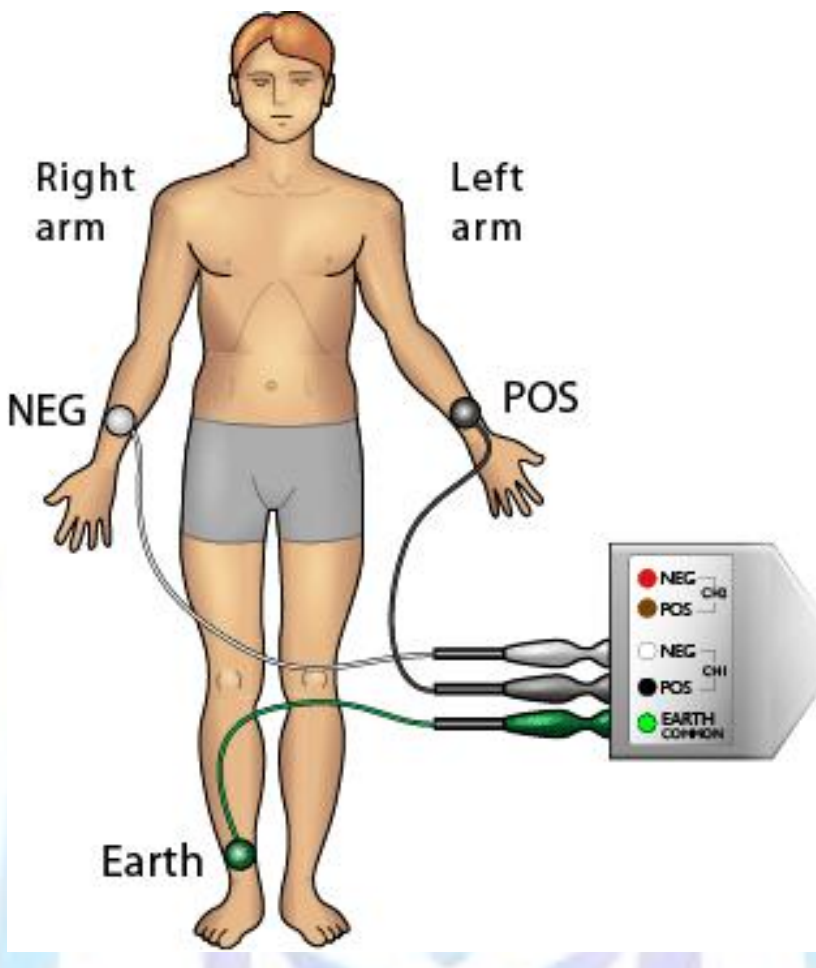

You will record and examine the major components of the Electrocardiogram (ECG).

\section{Procedure}

1. The subject should relax and sit as still as possible to minimize signal artifacts due to movements.

2. Type the subject's name into the Comment panel.

3. Click Start, then add the comment.

Click Autoscale as required to ensure that you can see all the data as it is being recorded.

4. If the ECG cannot be seen, check that all three electrodes are correctly attached. If the signal is noisy and indistinct, make sure that the subject is relaxed; consider using the alternative attachment positions.

5. Click Stop.

6. Click Start again. While recording, ask the subject to open and close his or her hands, and then move both arms across the chest.

The trace moves all over the place, and the ECG becomes distorted. This shows you why it is necessary for subjects to keep still and stay relaxed while their ECG is being recorded.

7. With the subject sitting quietly, click Start again. When you have a trace without movement artifacts, type 'Resting ECG ' and the subject's name, and add the comment.

8. Click Stop.

\section{Analysis}

1. Scroll through your data and observe the regularly occurring ECG cycles.

2. In a representative cycle, measure the amplitudes and durations of the $\mathrm{P}$ wave, $\mathrm{QRS}$ complex and T wave.

3. To measure the amplitudes, place the Marker on the baseline immediately before the $P$ wave. Then move the Waveform Cursor to the peak of a wave. Click to place the number in the Value panel. 
4. Drag the number from the Value panel into the appropriate column of the upper table.

5. To measure the durations, leave the Marker at the start of the wave or complex and position the Waveform Cursor at the end of the wave or complex.

6. Click to place the number in the Value panel and then drag the number from the Value panel into the appropriate column of the table.

7. Now investigate how the heart rate may vary from beat to beat. To do this, set the horizontal compression to 10:1. Measure the time interval (in seconds) between three pairs of adjacent $\mathrm{R}$ waves using the Marker and Waveform Cursor.

8. Record your results in the lower table. For each interval, the heart rate is shown in column 3 of the table, calculated using the equation $\mathbf{H R}=\mathbf{6 0} \div \mathbf{t}$, where $\mathrm{HR}=$ Heart Rate (beats/min) and, $\mathrm{t}=$ time interval (seconds).

\section{Exercise 1: ECG at rest}

\section{Study Exercise}

Use the Comments panel to indicate on the recording the P-wave, the QRS complex, and the T-wave.

\section{Study Questions}

1. What can you say about the amplitude of the various waves in different cardiac cycles?

2. The $P$ wave and the QRS complex represent depolarization of the atrial and ventricular muscle respectively. Why does the QRS complex have the largest amplitude?

\section{Exercise 2}

In this exercise, you will record the resting ECG signal from other members of your group.

\section{Procedure}

1. Attach the electrodes to the new subject.

2. With the subject sitting quietly, click Start again. When you have a trace without movement artifacts, type 'Resting ECG' and the subject's name and enter the comment.

3. Click Stop.

4. Repeat for all other students in the group

\section{Analysis}

Compare the duration and amplitude of the $\mathrm{P}$ waves, QRS complexes and T waves between those in your group and with other members of the class. Record your results in the Table.

\section{To Measure Waveform Amplitude:}

1. Drag the Marker to the lowest point of the waveform before each of the peaks of interest.

2. Move the Waveform Cursor to the peak, to the right of the Marker, and click.

3. Drag the number from the ECG Value panel into the appropriate Amplitude column of the table.

\section{To Measure Waveform Duration:}

1. Leave the Marker on the lowest point of the waveform before a peak.

2. Move the Waveform Cursor to the lowest point following the waveform, and click.

3. Drag the number from the Time Value panel into the appropriate column of the table.

\section{Exercise 2: ECG variation}

\section{Study Questions}

1. Are the amplitudes and durations of the various waves in different individuals similar or very different?

2. What variations in heart rate did you observe between individuals?

\section{Exercise 3}

You will measure and correlate the ECG and heart sounds in a resting volunteer. 


\section{Using a Stethoscope}

The stethoscope bell is better than the diaphragm for this exercise because it blocks off room noise. It still helps if everyone tries to keep the noise down.

Your instructor will briefly demonstrate how to use the stethoscope.

The volunteer should place the bell of the stethoscope on the left side of their chest, using the right hand. (It is easy enough to do this under one's shirt.) The stethoscope should be moved to different positions until the student listening to the stethoscope hears clear heart sounds. The sounds are soft, and room noise must be kept low. Once clear heart sounds are heard, the volunteer should hold the stethoscope in place with the right hand while the student listening to the stethoscope listens and records.

1. Click Start to record the ECG, and press the push-button switch on hearing 'lub' and release it on 'dub'.

2. After a few heart beat cycles, click $\underline{\text { Stop. }}$.

\section{Analysis}

To make it easier to compare the recordings in the two channels, the LabTutor panel has been set up to display the recordings overlaid. With the Channel Trace buttons you can select which of the two channels is 'active' in the panel.

1. Note the correlation between Event and ECG signals.

2. Using the Marker and Waveform Cursor, follow the instructions below to measure the time between the peak of the $\mathrm{R}$ wave and the Event signal going high.

1. Select the ECG channel as active

2. Place the Marker on the $\mathrm{R}$ wave

3. Select the Event channel as active

4. Use the waveform cursor and select the Event signal going high

5. Insert this time into the table

3. Now measure the time between the peak of the T wave and the Event signal going low.

1. Select the ECG channel as active

2. Place the Marker on the T wave

3. Select the Event channel as active

4. Use the waveform cursor and select the Event signal going low

5. Insert this time into the table

\section{Exercise 3: ECG and heart sounds}

\section{Study Questions}

1. Explain why ventricular contraction (systole) and the 'lub' sound occur immediately after the QRS complex.

\section{Explain why ventricular relaxation (diastole) and the 'dup' sound occur after the T} wave.

\section{Exercise 4}

You will record and correlate the ECG and heart sounds (with a cardiomicrophone) in a resting volunteer.

Clearly, the method that you used in Exercise 3 is subject to considerable error. For example, reaction time will introduce a significant delay.

The alternative, phonocardiography, is to use a microphone placed over the chest wall to record the heart sounds which can then be displayed graphically in real time.

\section{Procedure}

1. Unplug the push button from Input 1 and plug the cardiomicrophone into Input 1.

2. Place the cardiomicrophone on the left side of your chest. Then hold it firmly in place either by a strap running around the chest or by placing a heavy book or similar object on top of it. (This requires that you lie down). 
It is essential that the microphone is not held onto the chest-wall by hand, as the inevitable movement of the hand introduces considerable noise into the recording.

3. Click Start to record the ECG and cardiomicrophone signals. You should try placing the microphone in different positions to get the best possible signal.

4. After about 15 seconds, click $\underline{\text { Stop }}$

\section{Analysis}

Again, to make it easier to compare the recordings in the two channels, the LabTutor panel is set up so that the recordings are overlaid.

Clicking the buttons towards the top right of the panel allows you to select which of the two channels is 'active' in the panel.

1. Note the relationship between the R-wave and the first sound. Using the Marker and Waveform Cursor, follow the instructions below to measure the time between the peak of the $R$ wave and the beginning of the first heart sound.

1. Select the ECG channel as active.

2. Place the Marker on the $R$ wave.

3. Select the PCG channel as active.

4. Use the waveform cursor and select the beginning of the first heart sound.

5. Insert this time into the table.

2. Note the relationship between the T-wave and the second sound. Now measure the time between the peak of the T wave and the beginning of the second heart sound by repeating steps 1 to 5 above

\section{Exercise 4: ECG and phonocardiography}

\section{Study Question}

1. Your "lub-dub" recordings probably show some differences from the correct timing of the heart sounds as judged by phonocardiography. How can you account for this difference?

\section{Experiment 4 ElectroCardioGraph and peripheral circulation}

\section{Introduction}

The heart is a dual pump that pushes blood around the body and through the lungs. The beating of the heart results in a blood flow that is itself rhythmic. In this laboratory, you will measure the finger pulse and correlate it with the ECG. In addition you will palpate various arteries, and look at the peripheral circulation in the hand and the effects of cold.

\section{Learning Objectives}

By the end of today's laboratory you will be able to:

- Measure the ECG and pulse at rest

- Analyze the resultant signals, and look at the variations

- Palpate the radial, ulnar and brachial pulses

- Show that the arterial blood supply to the fingers derives from both radial and ulnar arteries by way of anastomoses

- Measure the effect of cold on the amplitude of the finger pulse 


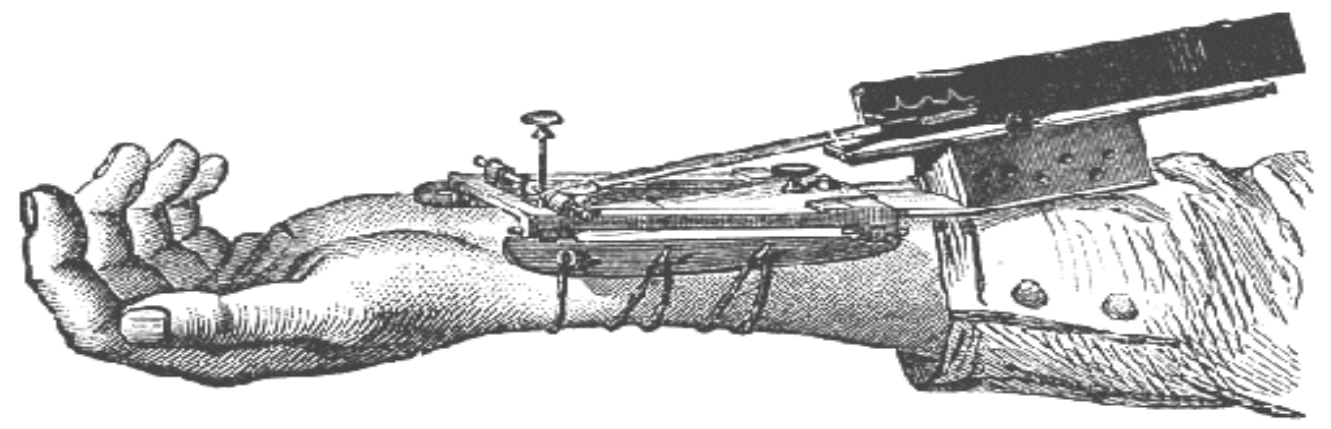

An early system for recording the human pulse, the Direct Sphygmograph (after Etienne-Jules Marey

\section{Procedure}

1. Make sure the PowerLab is connected and turned on.

2. Attach the Finger Pulse Transducer to a middle finger.

3. Connect the Finger Pulse Transducer to Input 1.

4. Remove any watches and/or jewelry from your wrists and ankles.

5. Connect the electrode lead wires to Earth, and CH1 NEG, and POS on the Bio Amp cable.

6. Plug the Bio Amp cable into the Bio Amp input.

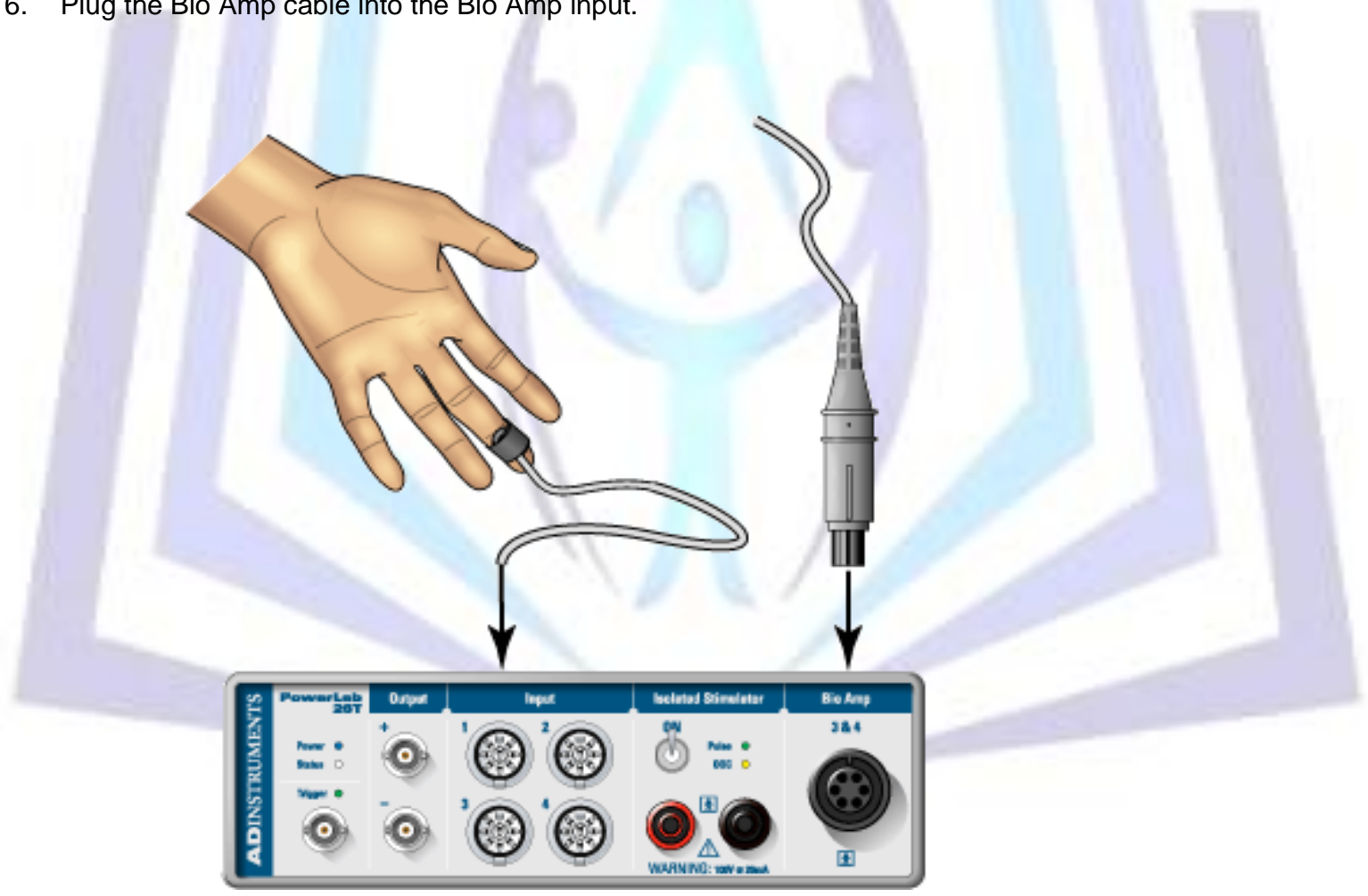

\section{Standard Connection}

Attach the positive electrode to the left wrist, the negative to the right wrist, and the ground to the right leg.

1. Using a pen, mark each point where electrodes will be placed. Clean the skin with alcohol swabs and lightly abrade the area with abrasive gel or a pad. This reduces the electrical resistance of the outer layer of skin and ensures good electrical contact.

2. If you are using the Reusable Clamp Electrodes, apply a small amount of electrode cream to the electrodes before attaching. Electrode cream is not necessary if you are using disposable electrodes which have electrode gel on them already.

3. If, after looking at the signal during the first exercise, you find that this does not produce a good signal, try the alternative method. 


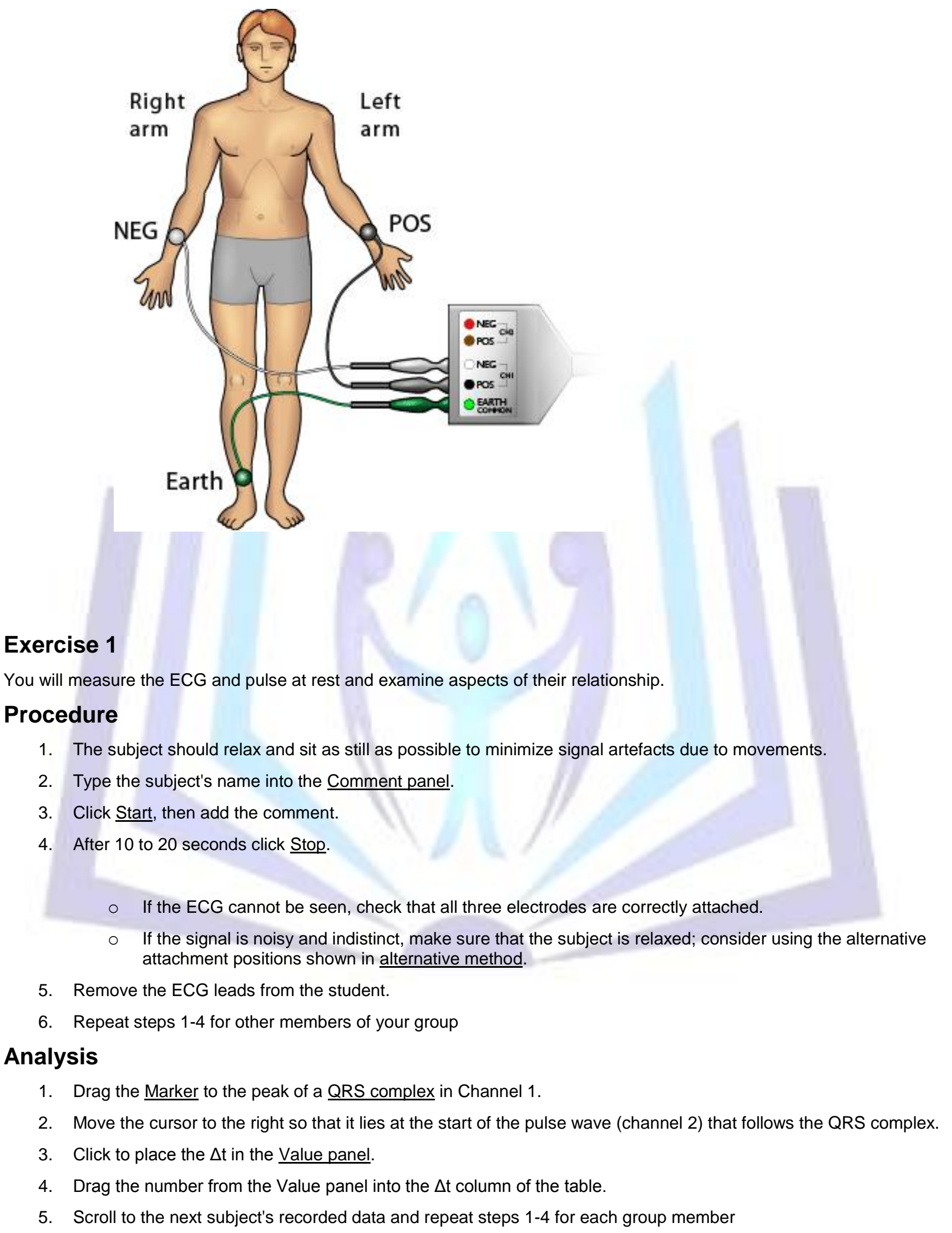




\section{Exercise 1: ECG and pulse at rest \\ Study Questions}

1. Today in class, you measured an electrical signal (ECG) produced by the heart. In your own words, describe as completely as you can the structures and processes that produces the QRS complex in the ECG you measured today.

2. List the physiological processes that take place between the QRS complex and the arrival of the pulse wave at the finger.

\section{Exercise 2}

You will measure the pulse in other students to determine the variation between individuals.

\section{Procedure}

1. Attach the Finger Pulse Transducer to the student.

2. Type the subject's name into the Comment panel.

3. Click Start.

4. Click Add to add the comment and record for 10 seconds.

5. Click Stop.

6. Repeat steps 1-5 for each member of your group

\section{Analysis}

Using the Marker and Waveform Cursor, determine the pulse amplitude and pulse interval. The heart rate for each member of your group will be automatically calculated and displayed in the table.

For each student, measure the pulse amplitude and interval between pulses.

\section{To measure pulse amplitude:}

1. Drag the Marker to the lowest point of the waveform before a peak.

2. Move the Waveform Cursor to the peak, to the right of the Marker, and click.

3. Drag the number from the Pulse Value panel into the Amplitude column of the table.

\section{To measure the interval between pulses:}

1. Drag the Marker to the highest point of a peak.

2. Move the Waveform Cursor to the peak to the right of the Marker, and click.

3. Drag the number from the Time Value panel into the Interval column of the table.

The last column of the table shows the heart rate calculated from the interval as $60 / \Delta t$.

\section{Exercise 2: The Pulse}

\section{Study Question}

1. Suggest some reasons for the differences in pulse amplitude from subject to subject.

\section{Exercise 3}

You will learn to palpate the main peripheral arterial pulses.

\section{Procedure}

1. Feel the subject's radial pulse, at the place indicated in the figure. You should feel it with the first three fingers, your index, middle and ring fingers, placed in a line along the length of the radial artery.

Don't use your thumb for palpation; it has a strong pulse in it, and you may end up feeling your own pulse instead of the subject's.

- Don't press too hard; only light to moderate pressure is needed to feel a pulse.

- When looking for the arteries, it is best to glide the fingers back and forth slowly over the region rather than immediately applying pressure.

2. Attempt to feel the subject's ulnar pulse. In most people, no pulse can be felt. 
3. Feel the brachial pulse at the elbow.

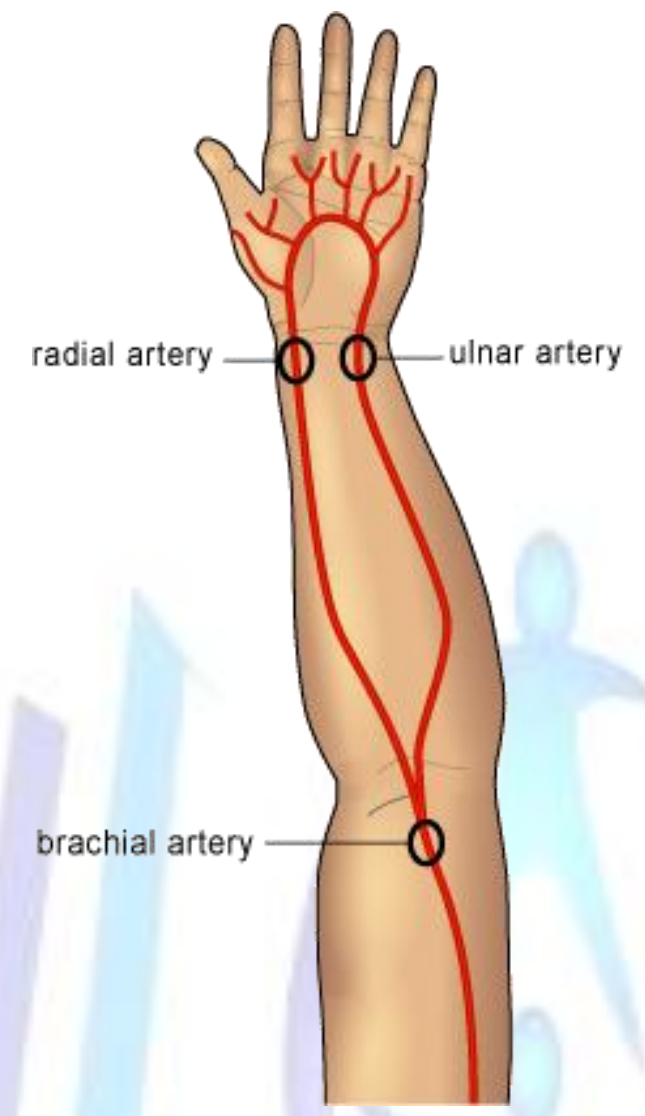

\section{Exercise 3: Palpation of arterial pulses}

\section{Study Questions}

1. When you feel a pulse, do you feel (a) the blood flow, (b) the pressure wave, or (c) brief changes in diameter of the artery due to the pressure wave?

2. Anatomical sites where a pulse can be palpated often correspond to 'pressure points' for stopping hemorrhage in first-aid treatment. Why?

3. Why can the ulnar pulse not usually be felt?

4. Doctors are trained to assess various aspects of a pulse: the rate, rhythm, amplitude and quality. For example, the rate might be 72 beats per minute, rhythm regular or irregular, amplitude full and quality 'thready' or collapsing. Based on your experiences in the lab today, which of these parameters do you think would be easy to assess, and which would be difficult?

\section{Exercise 4}

This exercise demonstrates that the arterial blood supply to the fingers derives from both radial and ulnar arteries by way of anastomoses (connections between the vessels) in the hand.

\section{Procedure}

1. Place the Finger Pulse Transducer on the distal segment of your middle finger as before.

2. Click Start.

3. Now, for the brachial, radial and ulnar arteries in turn, do the following:

- Apply firm pressure with the ball of the thumb over the artery for 5-10 seconds, and then release it.

- Add a comment with the artery name to the recording when you start to apply the pressure and the comment "release" when you release the pressure.

4. Click Stop and observe your data. The waveforms should look something like those in this figure 


\section{Analysis}

The raw recorded data is shown in the top channel.

The bottom channel shows the pulse amplitude computed from the raw data (as the peak to peak excursion in each pulse cycle).

Carefully examine your data, to see what effect compression of each artery had on the finger pulse.

\section{Exercise 4: Arterial anastomoses in the hand}

\section{Study Questions}

1. In your own words, describe why the pulse in the fingers disappeared when the brachial artery was compressed?

2. Did the pulse disappear completely when the radial or ulnar artery alone was compressed? If not, why not?

3. There is much anatomical variation from person to person, but in most people, blood flow to the fingers is derived mainly from the ulnar artery, with a smaller contribution from the radial artery. On the basis of your results, does the subject seem to conform to the general rule?

\section{Exercise 5}

You will examine the effect of cold on the amplitude of the finger pulse.

\section{Procedure}

1. Click Start.

2. Record $10-20$ seconds of data with the subject's hand at its normal temperature.

3. Click Stop.

4. Add a comment 'before cold' near the end of the data you just recorded.

5. Remove the Finger Pulse Transducer from the hand.

6. Immerse the subject's hand in a basin of ice-water for 30 seconds, or until the subject feels too much discomfort. The greater the immersion time, the larger the difference you will be able to observe.

7. Dry the hand and re-attach the Finger Pulse Transducer.

8. Click Start.

9. Add a comment 'return to warmth' near the start of the data you are recording now.

10. Record for several minutes during the pulse recovery as the hand warms up.

11. Click Stop.

Click Autoscale as required to ensure that you can see all the data as it is being recorded.

\section{Analysis}

1. Using the Marker and waveform cursor, note the amplitude in channel 2 of a representative pulse-beat every thirty seconds over the duration of your recording.

2. Transfer these values from the Value panel into the table.

\section{Exercise 5: The effect of cold on the pulse}

\section{Study Questions}

1. In your own words, describe the effect cold had on the pulse.

2. Many mammals have the ability to restrict blood flow to the extremities in cold environments. Does your data support this general observation?

\section{Experiment 5}

\section{ElectroEncephaloGraphy (EEG)}

\section{Introduction}

In this experiment, you will record and analyze electroencephalograms (EEGs) from a volunteer to look at the effect of various interfering signals. You will examine the effect on alpha and beta waves caused by opening and shutting the eyes, as well as auditory and mental cues. 


\section{Learning Objectives}

By the end of today's laboratory you will be able to:

- Recognize common artifacts in EEG signals.

- Identify and quantify alpha waves.

- Understand how alpha wave activity is changed by mental activity and auditory stimulation

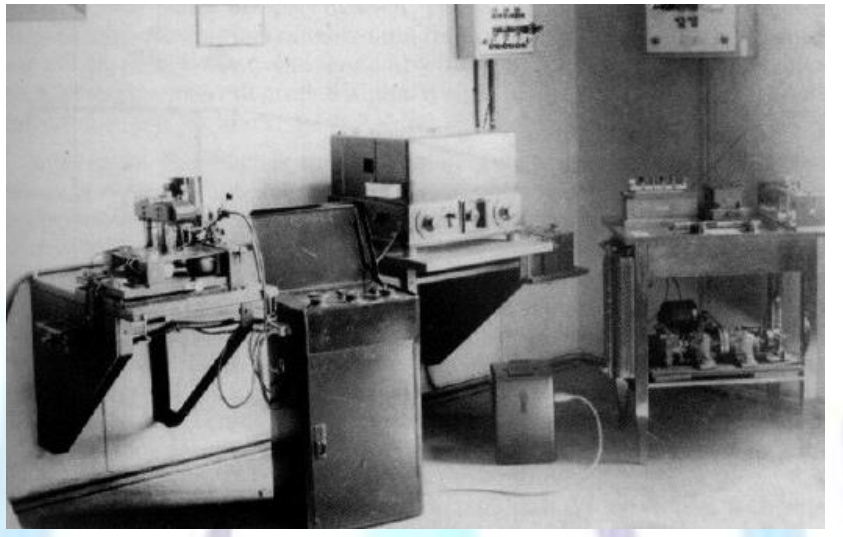

Berger's first EEG recording equipment

\section{Equipment setup}

- Plug the Bio Amp cable into the Bio Amp socket on the PowerLab.

- Connect the leads of three EEG flat electrodes to Earth, CH1 NEG and POS, on the Bio Amp cable.

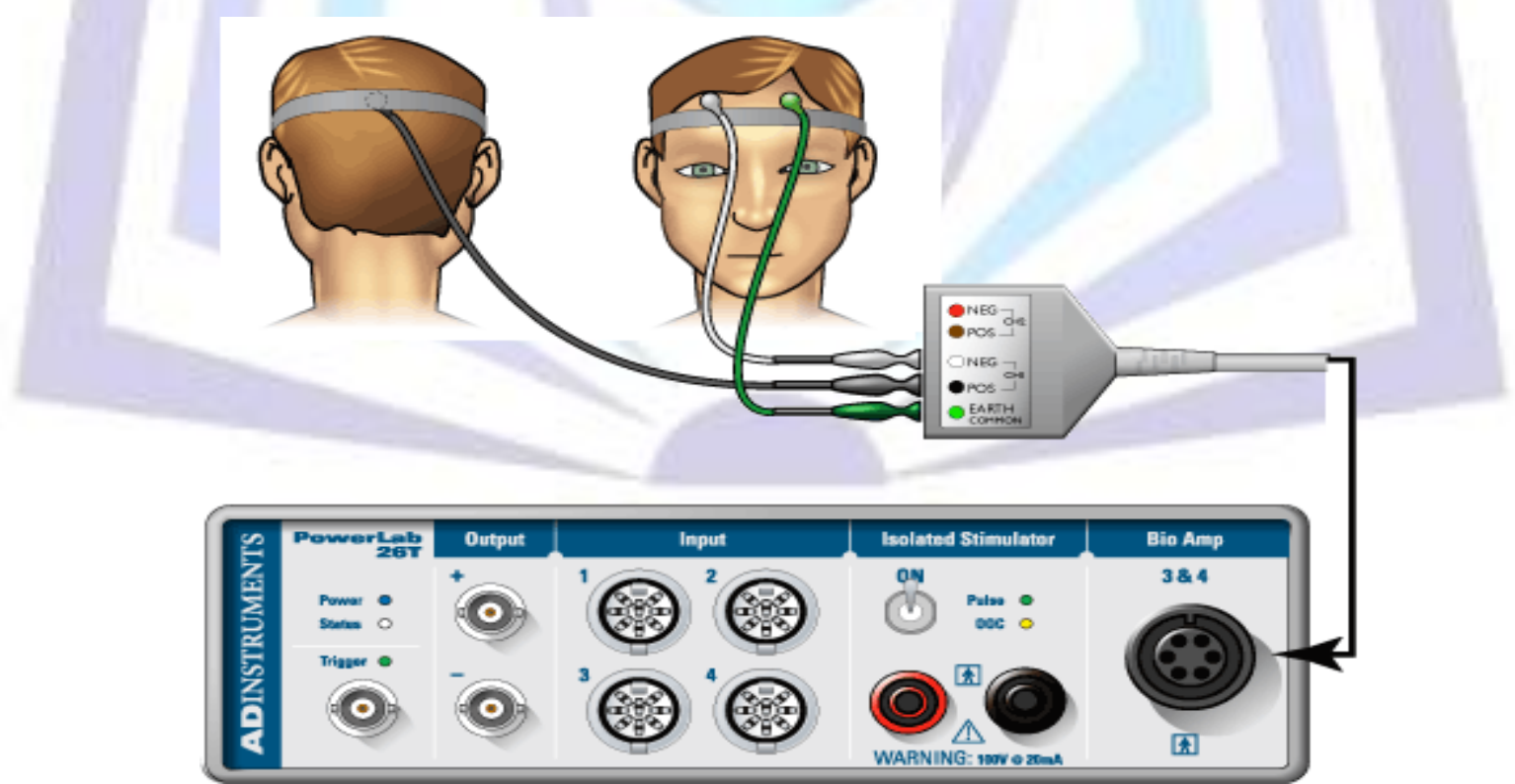

Exercise 1: Recognizing Artifacts

The top channel in the LabTutor panel shows the raw EEG, the lower 4 channels display digitally filtered raw data:

- Alpha frequency (8 to $13 \mathrm{~Hz}, \sim 30-50 \mu \mathrm{V}$ )

- Beta frequency $(13$ to $30 \mathrm{~Hz},<20 \mu \mathrm{V})$

- $\quad$ Theta frequency $(4$ and $8 \mathrm{~Hz},<30 \mu \mathrm{V})$ 
- Delta frequency $(0.5$ and $4 \mathrm{~Hz}, \sim 100-200 \mu \mathrm{V})$.

During the experiment, use the scrollbars, Autoscale and Compression controls whenever necessary to ensure you can see all the data recorded.

Remember to ensure that the volunteer is relaxed and lies still except when instructed otherwise.

\section{Blinking Artifact}

1. Click Start. Ask the volunteer to blink repeatedly.

2. Watch the volunteer and push the enter key to enter a comment each time the volunteer blinks.

3. After 5 to 10 seconds, click Stop.

\section{Eye Movements}

1. Click Start. Ask the volunteer to gaze alternately left then right, in a repeated pattern. The volunteer should keep the head still during these movements.

2. Watch the volunteer and push the enter key to enter a comment each time their eyes move.

3. After 5 to 10 seconds, click $\underline{\text { Stop }}$

\section{Head Movements}

1. Click Start. Ask the volunteer to move their head alternately left then right, in a repeated pattern.

2. Watch the volunteer and push the enter key to enter a comment each time their head moves.

3. After 5 to 10 seconds, click $\underline{\text { Stop. }}$.

\section{Analysis}

1. Examine the recordings using the scroll bar at the bottom of the LabTutor panel and by adjusting the vertical scale.

2. True EEG signals rarely exceed $+50 \mu \mathrm{V}$ and $-50 \mu \mathrm{V}$. You should find large signals outside the $\pm 50 \mu \mathrm{V}$ range that relate to blinking, eye movements and head movements. These large signals are artifacts. If you do not see such signals, check the electrode connections, and if necessary, remove and re-attach any connections that seem of dubious quality.

There are three common causes of artifacts such as those you have recorded:

1. Electromyographic (EMG) activity in muscles of the face or scalp (e.g., blinking, head movements).

2. Potentials arising from rotation of the eyes (electro-oculographic or EOG signals).

3. Mechanical movement of electrodes, especially the occipital one, whose attachment is made insecure by hair.

\section{Exercise 1: Recognizing Artifacts}

Using the Horizontal Compression buttons and the scroll bar, display one example of an artifact in your EEG recording.

\section{Study Question}

1. Name three sources of artifacts in EEG recordings, and in which waveform these artifacts are most prevalent.

\section{Exercise 2: Alpha \& Beta Rhythm}

This part of the experiment examines alpha and beta waves (alpha or beta rhythm) in the EEG, and the effect of having the eyes open or shut on this rhythm.

\section{Procedure:}

1. Ensure that the volunteer is relaxed, lying quietly and has both eyes open.

2. Click Start.

3. Type 'shut' in the Comments panel. After about 30 seconds, ask the volunteer to shut both eyes. Immediately click Add to enter the comment and continue recording.

4. Type 'open' in the Comments panel. After about 30 seconds, ask the volunteer to open both eyes. Immediately click Add to enter the comment.

5. Record for a few more seconds then click Stop.

6. Repeat this procedure twice more to give you three sets of results. 


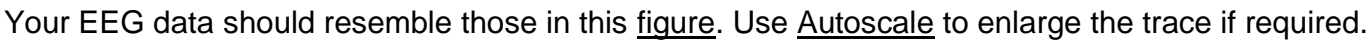

\section{Analysis}

Using the Horizontal Compression buttons and the scroll bar, review your recording looking for evidence of a change in alpha waves amplitude between eyes open and eyes shut. Look also for any change in beta wave activity.

You can recognize alpha waves by their amplitude and their timing. They are usually less than $50 \mu \mathrm{V}$ (although it can be quite variable from volunteer to volunteer). Each cycle of an alpha wave should last almost exactly $0.1 \mathrm{~s} \underline{\text { as in this }}$ example.

- If you cannot find evidence of a change in alpha wave activity between eyes open and eyes shut, check that you are examining records taken with the volunteer's eyes shut.

- If your records consist mainly of large-amplitude artifacts, you may need to re-attach one or more electrodes.

\section{Some volunteers may not exhibit significant alpha wave}

\section{Exercise 2: Alpha \& Beta Rhythm}

Using the Horizontal Compression buttons and the scroll bar, display the data for Exercise 2 you wish to include in your report.

\section{Study Questions}

\section{Under what conditions did you see alpha waves more clearly? \\ 2. What are alpha waves thought to indicate?}

\section{Exercise 3: Effects of Mental Activity}

In this exercise you will examine the effect of a mental arithmetic task on alpha and beta rhythm, while the eyes are closed.

\section{Procedure}

1. Ensure that the volunteer is relaxed, lying quietly and has both eyes closed.

2. Click Start and enter the comment 'shut'.

3. After 30 seconds or so of sustained alpha rhythm give the volunteer the following instructions:

- On your instructions, start mentally subtracting multiples of 7 from an arbitrary starting number e.g.; $100-7=93,93-7=86,86-7=79$, etc...

Instruct the volunteer to merely think the response and not speak it.

4. Add the comment 'math' when you instruct them to start the mental arithmetic.

5. After another thirty seconds or so of the mental arithmetic instruct the volunteer to stop subtracting and to relax.

6. Record for a few more seconds then click $\underline{\text { Stop. }}$.

7. Repeat this procedure twice more to give you three sets of results. Vary the mental arithmetic task for each repetition

\section{Analysis}

Identify the alpha and beta waves associated with eyes shut with no arithmetic (control) and when the mental arithmetic started. Now repeat the analysis as for Exercise 2.

1. From your first recording, select a portion when the volunteer's eyes were shut but they weren't performing any mental arithmetic.

2. Drag the calculated measurements of amplitude and frequency from the Value panels into the appropriate cell in the tables below.

3. Repeat these steps for all three recordings of eyes shut, with and without arithmetic. The tables will display average amplitude and frequency, along with standard deviation (SD).

4. Once you have completed the analysis use the navigation buttons below the tables to view a graph of these variables.

The graph generated by LabTutor shows the percentage change in both amplitude and frequency between the no arithmetic (control) and arithmetic conditions for each waveform. (No arithmetic $=100 \%$ ). 


\section{Exercise 3: Effects of Mental Activity}

Using the Horizontal Compression buttons and the scroll bar, display the data for Exercise 3 you wish to include in your report.

\section{Study Questions}

1. What effects did the mental arithmetic have on the alpha wave activity?

2. How do you account for your findings?

\section{Exercise 4: Effects of Auditory Stimulation}

In this exercise you will examine the effect of different types and volumes of music on alpha and beta rhythm, while the eyes are closed.

\section{Procedure}

1. You will need a set of headphones and a method of playing music to the subject.

2. Cue the following types of music for presentation to the volunteer.

- Soothing (classical) music, low volume.

○ Soothing (classical) music, high volume.

- Intense (rock) music, low volume.

Intense (rock) music, high volume.

3. Ensure that the volunteer is relaxed, lying quietly, has the headphones on and has both eyes closed.

4. Click Start and enter the comment 'shut'.

5. After 30 seconds or so of sustained alpha rhythm present the first type of music and add an appropriate comment

6. After another 30 seconds or so of recording stop the music.

7. Record for a few more seconds then click $\underline{\text { Stop. }}$.

8. Repeat this procedure for each different type and volume of music.

\section{Analysis}

Identify the alpha and beta waves associated with eyes shut (control) and each type of music presented. Now repeat the analysis as for Exercise 2 and 3.

1. From your first recording, select a portion when the volunteer's eyes were shut with no music playing.

2. Drag the calculated measurements of amplitude and frequency from the Value panels into the appropriate cell in the tables below.

3. Repeat these steps for all recordings of eyes shut, with and without the different types of music. The tables will display average amplitude and frequency.

4. Once you have completed the analysis use the navigation buttons below the tables to view a graph of these variables.

The graph generated by LabTutor shows the percentage change in both amplitude and frequency between the no music (average) and the different types of music, for each waveform. (No music $=100 \%$ ).

\section{Exercise 4: Effect of Auditory Stimulation}

Using the Horizontal Compression buttons and the scroll bar, display the data for Exercise 4 you wish to include in your report.

\section{Study Questions}

1. What effects did the different types of music have on the alpha wave activity?

2. How do you account for your findings?

\section{Experiment 6 \\ ElectroMyoGraphy (EMG)}




\section{Introduction}

In this laboratory, you will explore the electrical activity of skeletal muscle by recording an electromyogram (EMG) from a volunteer. You will examine the EMG of both voluntary and evoked muscle action, and measure nerve conduction velocity.

\section{Learning Objectives}

By the end of today's laboratory you will be able to:

- Record EMG during voluntary muscle contractions, and investigate how contractile force changes with increasing demand

- Examine the activity of antagonist muscles and the phenomenon of coactivation

- $\quad$ Record EMG responses evoked by stimulating the median nerve at the wrist

- Measure nerve conduction velocity from the difference in latencies between responses evoked by nerve stimulation at the wrist and the elbow

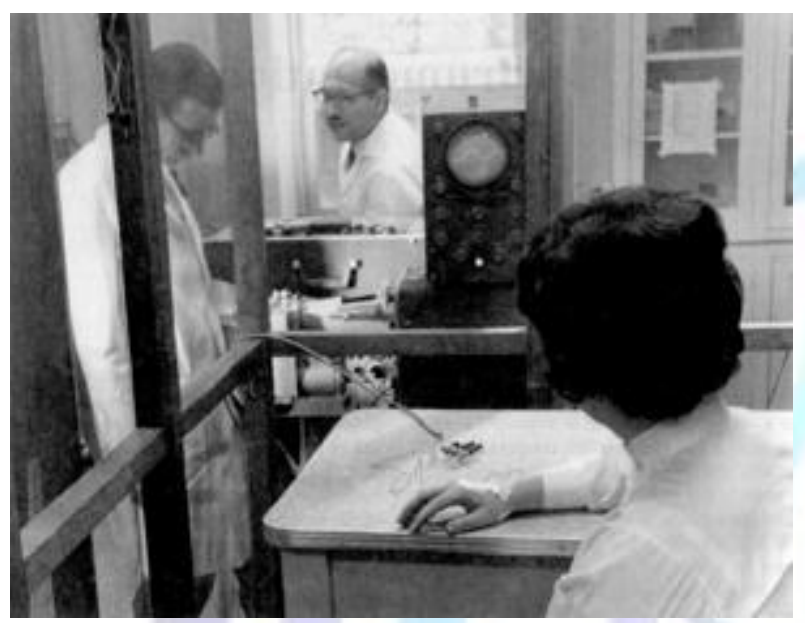

An early surface EMG recording.

John Basmajian, the pioneer of this technique, is in the middle of the photograph.

\section{Procedure}

\section{Caution!}

Some exercises involve application of electrical shocks to muscle through electrodes placed on the skin.

People who have cardiac pacemakers or who suffer from neurological or cardiac disorders should not volunteer for such exercises.

If the volunteer feels major discomfort during the exercises, discontinue the exercise immediately and consult your instructor.

\section{Electrode attachment:}

1. Remove any watch, jewelry, etc. from your wrists.

2. Plug the five-lead Bio Amp cable into the Bio Amp socket on the PowerLab unit.

3. Plug the five color-coded lead wires into the Bio Amp cable, as shown.

4. Firmly attach the dry earth strap around your palm or wrist. The fuzzy side of the dry earth strap needs to make full contact with the skin. Attach the green lead wire to the earth strap. If the dry earth strap has a single connector lead wire it should be inserted onto the pin nearest the Earth label.

5. If alcohol swabs are available, firmly swab the skin with them in each area where electrodes will be placed. Lightly mark two small crosses on the skin overlying the biceps muscle, in the position for the biceps recording electrodes, as shown. The crosses should be $2-5 \mathrm{~cm}$ apart and aligned with the long axis of the arm. Lightly abrade the skin at these areas with abrasive gel or a pad.

This is an essential step as it decreases the electrical resistance of the outer layer of skin and ensures good electrical contact. 
6. Prepare the skin over the triceps for attaching the electrodes as outlined in step 5 for the biceps. The position for the triceps recording electrodes is shown in the figure.

7. Prepare the disposable ECG electrodes by removing the backing film. Place the electrodes onto the skin over the crosses so they adhere well.

8. Plug the four shielded lead wires into the Bio Amp cable ports for positive and negative, $\mathrm{CH} 1$ and $\mathrm{CH} 2$.

9. Snap the lead wires from $\mathrm{CH} 1$ on the Bio Amp cable onto the electrodes on the subject's biceps.Snap the lead wires from $\mathrm{CH} 2$ on the Bio Amp cable onto the electrodes on the subject's triceps. It does not matter which is positive and which is negative.

10. Check that all four electrodes and the dry earth strap are properly connected to the volunteer and the Bio Amp cable before proceeding.

11. Make sure the PowerLab is connected and turned on.

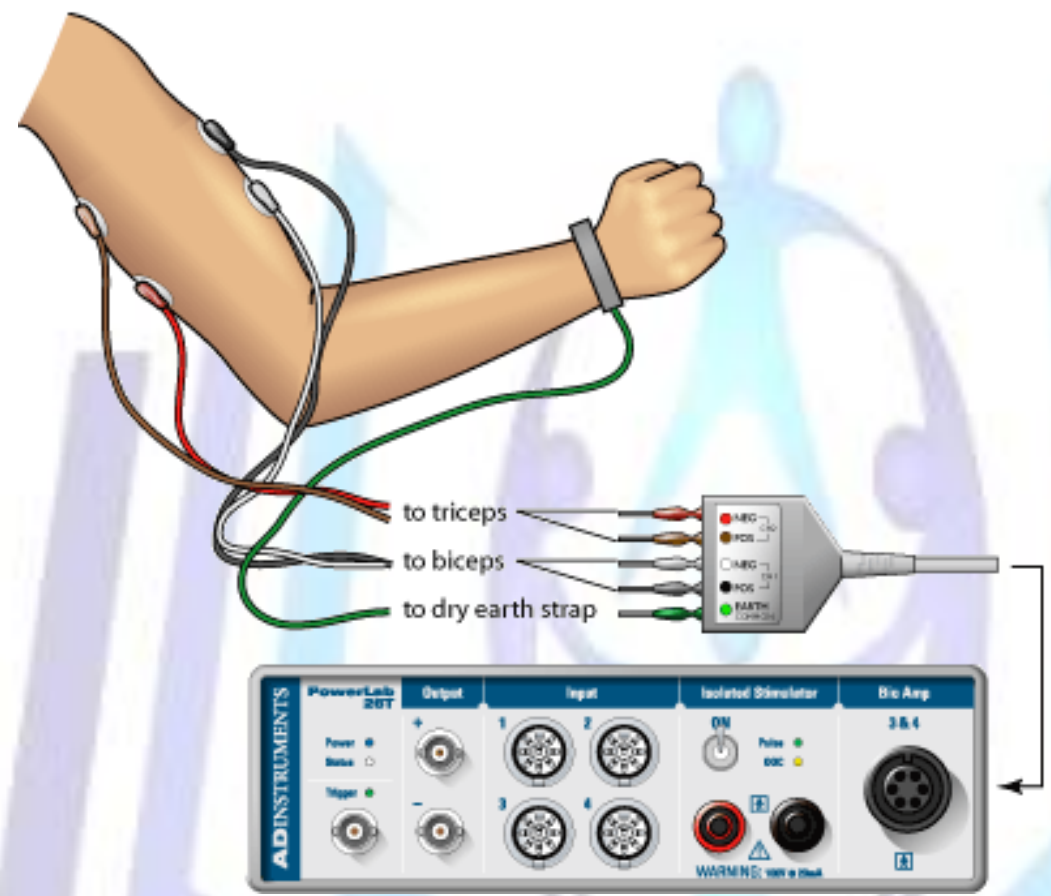

\section{Exercise 1}

You will record electrical activity during voluntary muscle contractions, and investigate how it changes with increasing demand.

The two lower channels in the LabTutor panel show raw activity; the two upper channels display integrated activity, calculated from the raw signal. Integrated activity is commonly used in the assessment of muscle function because it is easier to quantitate.

\section{Procedure}

During the experiment, use Autoscale whenever necessary to ensure you can see all the data recorded.

1. Sit in a relaxed position, with your elbow bent to $90^{\circ}$ and the palm facing upwards. Use your other hand to grasp the wrist of the arm from which the signal is being recorded.

2. Add a comment to the data file with your name.

3. Click Start.

4. Add the comment "biceps contraction", and immediately make a moderate contraction of the biceps muscle, by trying to bend the arm further and resisting this movement with your other arm. Observe the signal.

5. Enter the comment "triceps contraction", and immediately make a moderate contraction of the triceps muscle by trying to straighten out the arm and resisting this movement with your other arm.

6. Repeat steps 3 to 5 , but this time make a maximal contraction of the biceps and then the triceps muscles.

7. Click $\underline{\text { Stop. }}$. 
Remember to click Autoscale if the recorded signal traces are not all clearly visible in the LabTutor panel_.

8. Once again sit in a relaxed position, with your elbow unsupported and bent to $90^{\circ}$ with the palm facing upwards.

9. Click Start to resume recording.

10. Have someone place a book or a similar weight on your hand and add the comment "one book".

11. Leave the book in place for two to three seconds to record the change in the EMG.

12. Remove the book.

13. Click Stop.

14. Repeat steps 9-13 for two, then three, then four books, to give a series of increasing weights, adding a comment each time.

\section{Analysis}

1. Scroll through the recorded data and note the changes in activity in the raw biceps channel (Biceps). Note also that placing weights on the hand gives rise to little or no activity in the triceps muscle.

2. Choose a small part of the "Biceps" activity and examine it in more detail by setting the Horizontal compression to $1: 1$ and clicking Autoscale. Note that the raw EMG signal is composed of many partly-overlapping spikes.

3. Note the relationship between the raw trace (Biceps) and integrated trace (Int. Biceps). The height of the integrated trace reflects the overall activity of the raw EMG signal, and gives a simpler view of the muscle's electrical activity.

4. Use the Waveform Cursor and Value panel to record in the table the amplitude of the integrated trace as weights were added and removed. The height of the trace correlates with the force produced by the muscle.

\section{Exercise 1: Voluntary contraction}

\section{Study Questions}

1. Unlike the discrete waveform from an electrocardiogram, the electromyogram waveform is irregular. Why do you suppose this is?

2. In your own words, explain how the EMG trace changed when you added weights to your arm? Based on the data you collected what can you infer is happening to the muscles as weight is added?

\section{Exercise 2}

You will examine the activity of antagonist muscles and the phenomenon of coactivation.

\section{Procedure}

1. Sit in a relaxed position, with your elbow bent to $90^{\circ}$ with the palm facing upwards. Use the other hand to grasp the wrist of the arm from which the signal is being recorded.

2. Activate the biceps and triceps alternately as you did for Exercise 1. Practice this alternating pattern until it feels to you that both muscles are being equally activated in turn.

3. Click Start.

4. Perform the alternating pattern of activation for 20 to 30 seconds.

5. Click Stop.

6. Examine your data. The waveforms should look something like those shown here

\section{Analysis}

1. Scroll through the recorded data and observe the EMG traces for both the biceps and triceps.

2. Note the large-scale alternation of activity in the biceps and triceps.

3. Note that when the biceps muscle is activated forcefully, there is a minor increase of activity in the triceps. Correspondingly, there is a minor increase of activity in the biceps trace when the triceps are activated. This phenomenon is called 'coactivation'. Its physiological meaning is not well understood, although it perhaps serves to stabilize the elbow joint.

4. Measure and insert into the table the integrated EMG peaks for biceps and triceps during contraction of biceps and triceps. You do this using the two Value panels. 


\section{Procedure}

For this next exercise, you will stimulate the median nerve at the wrist and record muscle activity from the Abductor pollicis brevis (a thumb muscle). You can continue with the same volunteer or choose someone else.

Muscle contraction and sensations such as tingling or brief pain, are associated with nerve stimulation.

Since only two of the four recording electrodes are needed in this exercise, do the following:

- disconnect the recording electrode leads from the Channel 2 sockets of the Bio Amp cable,and remove their electrodes from the triceps of the subject

- remove the electrodes from the biceps of the subject, but leave their leads connected to the Channel 1 sockets of the Bio Amp cable.

Now continue with the setup:

1. With a ballpoint pen, lightly mark two small crosses on the skin above the abductor pollicis brevis muscle, in the position for the recording electrodes shown in the figure. The crosses should be $2-3 \mathrm{~cm}$ apart.

2. Lightly abrade the marked skin to reduce its electrical resistance.

3. Obtain two new disposable ECG electrodes and trim the adhesive pad slightly so they will fit as shown in the figure.

4. Attach the electrodes to the skin over the crosses you marked. To reduce electrode movement, use adhesive tape to attach the wires to the skin close to the electrode.

The dot on the back of the bar electrode indicates the positive electrode. Secure the electrode as shown in the figure, with the negative electrode closer to the wrist. The electrode should lie along the axis of the arm, with the leads pointing towards the hand.

5. Connect the Stimulating Bar Electrode to the Isolated Stimulator output of the PowerLab: the red (positive) connector to the red output and the black (negative) connector to the black output.

6. Place a small amount of electrode cream on the two silver pads of the stimulating bar.

7. Place the stimulus electrode over the volunteer's median nerve at the wrist (the approximate placing is shown in the figure).

8. Turn the Stimulator switch $\mathrm{ON}$. The Isolated Stimulator only becomes active during sampling; it is switched off internally at all other times.

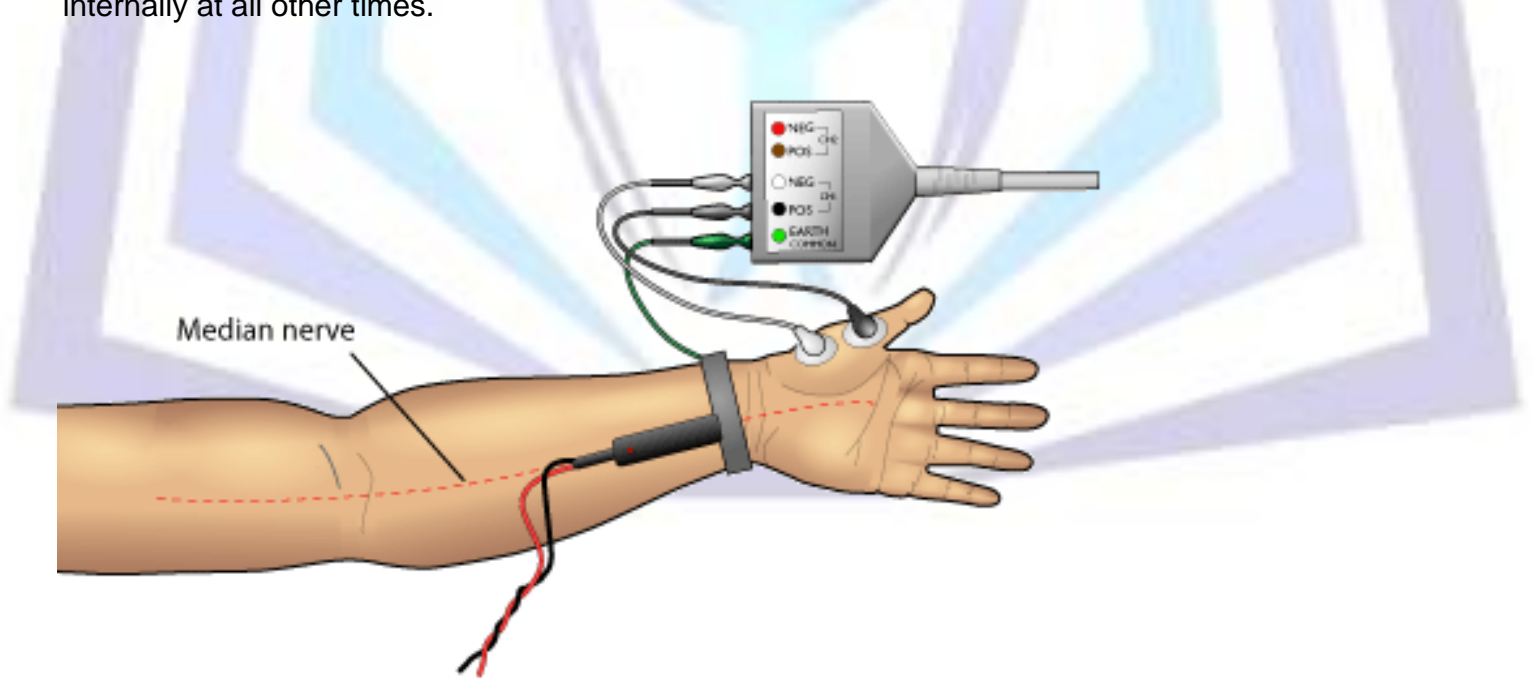

\section{Exercise 2: Alternating activity and coactivation}

\section{Study Questions}

1. In your own words what is coactivation? What are some explanations you can come up with for this phenomenon?

2. Coactivation of abdominal muscle and muscles supporting the spine has been shown to be essential for the bipedal posture of humans. Based on your data, is the coactivation of the triceps necessary for proper functioning of the biceps and vice versa? 


\section{Exercise 3}

You will stimulate the median nerve at the wrist and record muscle activity from the Abductor pollicis brevis (a thumb muscle).

\section{Procedure}

1. Set the pulse current in the Isolated Stimulator box to $8 \mathrm{~mA}$ by clicking the arrows or dragging the slider control. Recording will automatically stop after 0.05 seconds.

2. Click Start every time that you wish to stimulate. You should expect to see a waveform that looks something like this.

- Apply manual pressure to the back of the stimulus electrode to ensure that the nerve is stimulated and that the electrode doesn't move around during the exercise.

- $\quad$ Adjust the electrode to find the best position for stimulation as judged by the amplitude of the response.

- If you cannot get a response, increase the pulse current to 10 or even $12 \mathrm{~mA}$. If there is still no response try stimulating the ulnar nerve. (In some people, the abductor pollicis brevis is innervated by the ulnar nerve instead of the median nerve - an example of anatomical variation).

3. Once the electrode is optimally placed, increase the amplitude in $2 \mathrm{~mA}$ increments. Record the responses until either you reach $20 \mathrm{~mA}$ or the response no longer increases.

4. Turn the stimulator switch OFF.

5. Remove the stimulus electrode and mark with a pen the electrode indentation in the skin nearest to the hand.

\section{Analysis}

1. Use the scroll bar at the bottom of the LabTutor panel to review records recorded with stimulation at the wrist.

2. Measure the latency of a single waveform (the magnitude of the waveform is of no consequence).

'Latency' is the time elapsed from the start of the stimulus pulse (the start of each record) to the start of the evoked response. Note; you may see a very early deflection. This is the stimulus artifact and must be ignored.

3. Click at the point where the response begins.

4. Transfer the latency from the Value panel to the Latency (Wrist) column of the table. In the next exercise you will stimulate at the elbow and again measure the laten

\section{Exercises 3 \& 4: Evoked EMG \& nerve conduction velocity}

\section{Study Questions}

1. List the physiological events that occur between delivery of the stimulus and the start of the recorded response (i.e. during the latent period).

2. Which of the contributions to the latent period (that you listed in question 1 above) depends on the position of the stimulating electrode?

3. Based on your results and calculations for nerve conduction velocity, how long would it take for a nerve impulse to travel from the spinal cord to the big toe? Assume that the distance traveled is one meter.

4. Was there variability in the nerve conduction velocity amongst members of your group? What are some explanations for this?

\section{Exercise 4}

You will measure responses evoked by nerve stimulation at the elbow. The latency of these responses is longer than those evoked by stimulation at the wrist. You will be able to calculate nerve conduction velocity from the difference in latencies.

\section{Setup}

1. Position the Bar Stimulus Electrode on the medial aspect of the front of the elbow as shown here. The electrode requires firmer pressure at the elbow than at the wrist because the nerve is deeper in the tissues. The orientation of the electrode should be the same as for wrist stimulation, with the red dot positioned closest to the elbow.

2. Turn the Stimulator switch $\mathrm{ON}$. 


\section{Procedure}

1. Set the current in the Stimulator panel to $8 \mathrm{~mA}$.

2. Click Start every time you wish to stimulate. Do this several times, using these low-amplitude pulses to help to find the best position for the electrode.

3. If you cannot get a response, increase the stimulus current.

4. Once you have found the best position for the bar stimulus electrode, increase the stimulus to $15-20 \mathrm{~mA}$.

5. Click Start.

6. Repeat several times.

7. Turn the stimulator switch OFF.

8. Remove the stimulus electrode and mark with a pen the electrode indentation in the skin nearest to the hand. Remove the other electrodes.

\section{Analysis}

1. Measure and record the distance between the marks at the elbow and at the wrist. This is the distance between stimulation sites.

2. Use the same steps as outlined for wrist stimulation to measure the latency of a single waveform in the LabTutor panel.

3. Record the latency value in the table.

The conduction velocity is calculated automatically in the table, using the equation:

Velocity $=$ Distance $\div$ Time

The units of velocity are $\mathrm{mm} / \mathrm{ms}$ or, equivalently, $\mathrm{m} / \mathrm{s}$.

\section{Exercises 3 \& 4: Evoked EMG \& nerve conduction velocity}

\section{Study Questions}

5. List the physiological events that occur between delivery of the stimulus and the start of the recorded response (i.e. during the latent period).

6. Which of the contributions to the latent period (that you listed in question 1 above) depends on the position of the stimulating electrode?

7. Based on your results and calculations for nerve conduction velocity, how long would it take for a nerve impulse to travel from the spinal cord to the big toe? Assume that the distance traveled is one meter.

8. Was there variability in the nerve conduction velocity amongst members of your group? What are some explanations for this?

\section{Experiment 7}

\section{Electro- Oculo G raphy (EOG)}

\section{Introduction}

In this laboratory, you will record the electrical activity associated with eye movements: the electro-oculogram (EOG).

\section{Learning Objectives}

By the end of today's laboratory you will be able to:

- Describe the basis of the electro-oculogram

- Recognize common artifacts in EOG recordings and know their causes

- Describe the significance of angular displacement measurements

- Recognize saccades and understand their significance

- Record eye movements associated with smooth tracking

- Investigate aspects of gaze-holding and describe the significance of this concept

Professor Elwin Marg (1918 - ) whose seminal review," Development of Electro-Oculography", published in 1951, laid the foundations for the use of the EOG to study eye movmenents. 


\section{Procedure}

\section{Subject preparation}

1. Choose a member of your lab group as a subject.

2. Using a ballpoint pen, mark the areas on the skin for EOG electrode placement, as in Figure 1.

3. Lightly abrade the skin over the marks with abrasive gel or a pad.

4. Peel off the backing of one of the disposable, pre-gelled ECG electrodes and adhere it over one of the marked areas on the volunteer. Repeat for the other two electrodes.

5. Connect the lead wires to the subject with the snap-on connectors. Note that the wire from the forehead is the reference (earth) wire. Use the green lead for this connection.

6. Connect the three shielded lead wires to the rear of the EOG Pod. The green lead (earth) goes into the middle connector on the rear of the EOG Pod.

7. Plug the EOG Pod to the Input 1 of the PowerLab.

\section{Zeroing the EOG Pod}

1. Click Start.

2. Have the volunteer gaze on a point directly in front.

3. Using the knob on the front of the EOG Pod, zero the signal.

4. Click Stop.

The signal from the EOG Pod is prone to drift. You should check that the signal is zeroed before each exercise.

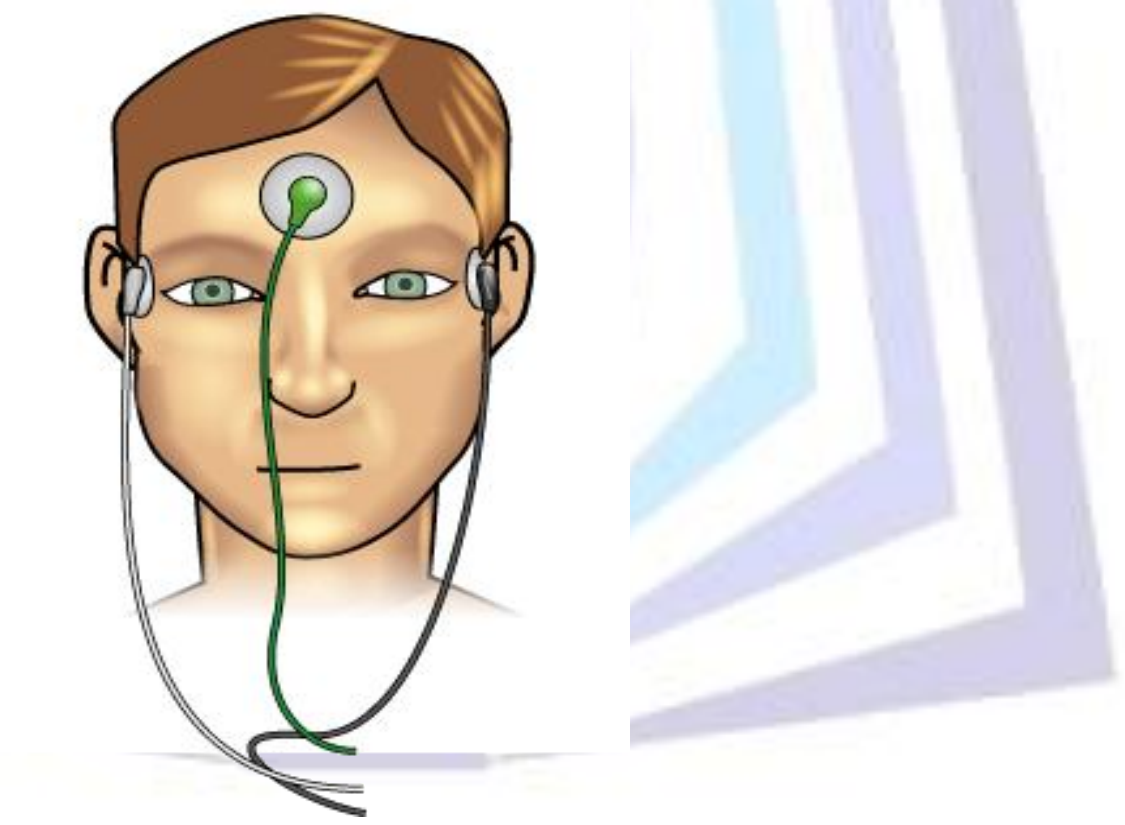

Figure 1. EOG electrode placement

\section{Exercise 1}

Because the EOG is recorded from the skin surface, it is essential to recognize signal artifacts and distinguish them from the actual EOG signal. Eye blinks are unavoidable; they will alter the EOG signal and it is important to be able to identify them. Electromyograph (EMG) signals from muscles in the face can also be recorded.

\section{Procedure}

1. Type in a comment: 'blink'. Do not add the comment yet.

2. Click Start.

3. Have the subject blink several times, and enter the comment. 

4. Click Stop.
5. Type in a comment: 'EMG'.
6. Click Start.
7. Have the subject clench the teeth together for several seconds.
8. Click ' Add' to add the comment to the Chart View.
9. Click Stop.

\section{Analysis}

Study the data to become familiar with these two artifact signals

\section{Exercise 1: Recognizing artifacts in the EOG}

\section{Study Questions}

1. Why do you suppose it is important to recognize artifacts in your data trace?

2. Briefly discuss why blinking would cause an artifact.

\section{Exercise 2}

In this exercise you will record angular displacement and relate it to EOG amplitude in a volunteer.

\section{Procedure}

1. Position a chair so that, when sitting comfortably, the subject's head is exactly one meter from the wall. Check the distance using a tape measure.

2. Make temporary marks on the wall at the subject's eye level with tape or chalk as shown here. These marks correspond to $+/-15^{\circ},+/-30^{\circ}$ and $+/-45^{\circ}$ angles at $1 \mathrm{~m}$ viewing distance.

3. Have the subject look at the center mark that is directly in front. This is 0 degrees. From now on, the subject's head should not move.

4. Type in a comment: 'angle calibration'.

5. Click $\underline{\text { Start }}$

6. Add the comment.

7. Ask the subject to move the eyes while keeping the head still and stare at the mark furthest to the left for two to three seconds. By convention angles to the volunteers left will be negative, angles to the right will be positive.

8. Add a comment: '-45'.

9. Repeat for the remaining marks on the wall, from left to right $(-45,-30,-15,0,15,30,45)$, adding an appropriate comment each time the subject views another point.

10. Click Stop.

\section{Analysis}

1. Place the Waveform Cursor on the portion of the trace corresponding to the $-45^{\circ}$ mark.

2. Click to place the amplitude of the EOG signal in the Value panel.

3. Drag the number from the Value panel into the amplitude column of the table.

4. Repeat these steps for each view angle.

\section{Exercise 2: EOG and angular displacement}

\section{Study Question}

1. Was the recorded EOG signal proportional to eye movement? In other words, is the response linear over the range of eye movement?

\section{Exercise 3}

Saccades are fast re-positioning movements of the eye. The easiest way to observe saccades is to record the EOG while the subject reads a page of text. 


\section{Procedure}

1. Type in a comment: ' fast tracking'.

2. Click Start and click Add to add the comment to the LabTutor panel.

3. Have the subject sit comfortably in front of the computer screen, ready to read a paragraph of text.

4. Click here to open a pop-up window which contains a paragraph of text of a suitable size for this experiment. It is essential to position this pop-up window over the LabTutor panel so that the subject is not distracted by the recording while reading the text. Alternatively, the subject may read from printed text provided to you by your Instructor.

5. Close the popup window.

6. Click Stop. Your data should look similar to this though it may be oriented in the other direction.

\section{Analysis}

1. Use the Horizontal Compression buttons to set a compression of 2:1.

2. Place the Marker at the start of a saccade. Each 'step' in the recorded signal corresponds to a single saccade.

3. Move the Waveform Cursor to the end of the saccade.

4. Click to add the duration of the saccade to the Value panel and drag the value to the appropriate table cell.

5. Select four more saccades and repeat steps 1-3 to get an average of five saccades in total.

\section{Exercise 3: Saccades}

\section{Study Questions}

1. When you observed saccadic eye movements during reading, what activity do you suppose correlated with the largest response?

2. Discuss the velocity of saccades in your recording. Why are saccades an important aspect of vision? Do you notice saccades when you are reading?

\section{Exercise 4}

In this exercise, you will examine the ability of the volunteer to follow a slowly moving object.

\section{Procedure}

1. Have a member of your group hold a pen or pencil about $50 \mathrm{~cm}$ in front of the subject who should be looking straight ahead.

2. Instruct the subject to fixate on the pencil without moving the head.

3. Type in a comment: 'smooth tracking'.

4. Click Start.

5. Add the comment.

6. Move the pencil slowly to the left and right of the subject keeping within a range that the subject can track with the eyes without moving the head.

7. Click Stop.

\section{Analysis}

1. Observe the recording.

2. Look for evidence of saccades

\section{Exercise 4: Smooth tracking}

\section{Study Question}

1. Did saccades occur during slow tracking? If so, why do you suppose that they occurred?

\section{Exercise 5}

So far we have examined aspects of gaze-shifting: the mechanisms that we use to enable us to see an object as clearly as possible. 
Gaze-Holding describes our attempt to track an object despite our own movements. This can involve both optokinetic and vestibulo-ocular reflexes. Head movements are detected far more rapidly by the vestibular apparatus than by the visual system. This can be demonstrated as follows:

\section{Procedure (i)}

1. Hold your hand at arm's length and wave it from side to side, gradually increasing the rate.

2. Now, hold your hand still but rotate your head from side to side, again gradually increasing the rate.

\section{Question}

1. Which was clearer, the image when your hand was moving, or the image when your head was moving?

\section{Procedure (ii)}

1. Time someone reading a long paragraph on a page in a book while shaking the head from side to side. Enter the time in the table.

2. Repeat this but with the head still and the page of the book moving from side to side. Enter this time in the table.

\section{Questions}

1. Describe what you observed when you moved your hand relative to your head and then your headrelative to your hand. What conclusions can you draw from this simple experiment?

2. Do the results in the table support your conclusion?

\section{Exercise 5: Gaze-holding}

1. Which was clearer, the image when your hand was moving, or the image when your head was moving?

2. Describe what you observed when you moved your hand relative to your head and then your head relative to your hand. What conclusions can you draw from this simple experiment?

3. Do the results in the table support your conclusion?

\section{Experiment 8}

\section{Muscle}

\section{Introduction}

In this experiment, you will explore how muscles work. You will electrically stimulate the nerves in the forearm to demonstrate recruitment, summation and tetanus; and will also examine some of the properties of muscle fatigue.

Guillaume DuChenne (de Boulogne) (1806-1875) was the pioneer of human muscle stimulation.

\section{Learning Objectives}

By the end of today's laboratory you will be able to:

- Demonstrate the effects of electrical stimuli using the nerves of the forearm

- Record and measure the muscular twitch response to nerve stimulation, and show recruitment in the twitch response as the stimulus strength increases

- Measure the effects of changing the interval between paired stimulus pulses and observe a short tetanic contraction

- $\quad$ Calibrate a hand dynamometer with respect to a volunteer's maximal grip strength

- Measure the decline in maximal force during a sustained contraction, and examine some properties of muscular fatigue 


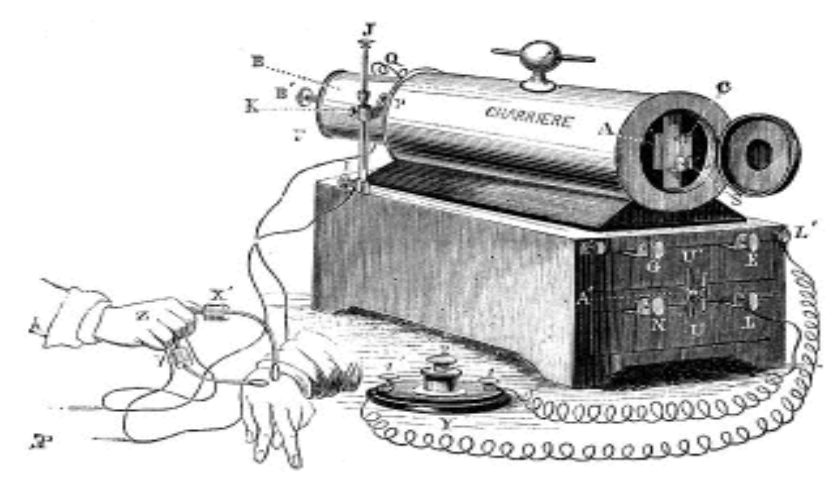

DuChenne's original equipment for stimulating human muscles.

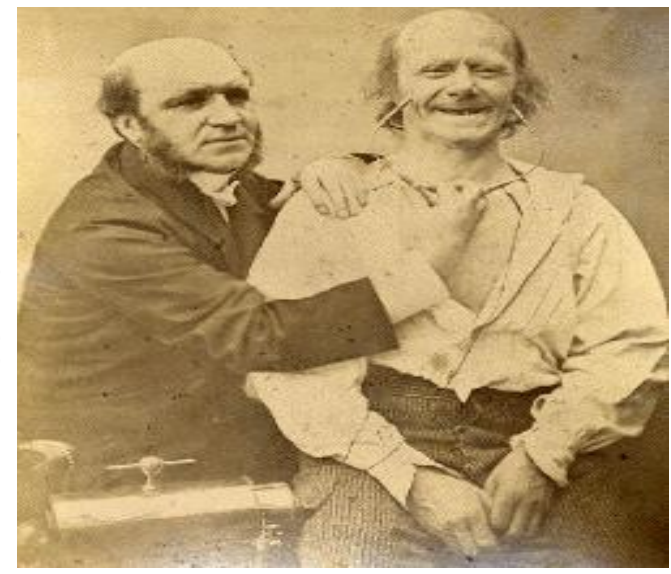

DuChenne using this equipment to stimulate facial muscles producing a 'smiling' contraction.

\section{Equipment Setup}

\section{Caution!}

Some exercises involve application of electrical shocks to muscle through electrodes placed on the skin.

People who have cardiac pacemakers or who suffer from neurological or cardiac disorders should not volunteer for such exercises.

If the volunteer feels major discomfort during the exercises, discontinue the exercise immediately and consult your instructor.

\section{Procedure}

1. Make sure the PowerLab is connected and turned on.

2. Connect the Finger Pulse Transducer to Input 1 on the PowerLab.

3. Place the Finger Pulse Transducer diaphragm-side up on the top of the lab bench, and tape the transducer in place along the Velcro strap.

4. Connect the Stimulating Bar Electrode to the isolated stimulator output of the PowerLab.

The leads are color-coded. Plug the red lead into the red socket and the black lead into the black socket.

5. Place a small amount of electrode cream on the two silver contacts of the stimulating bar.

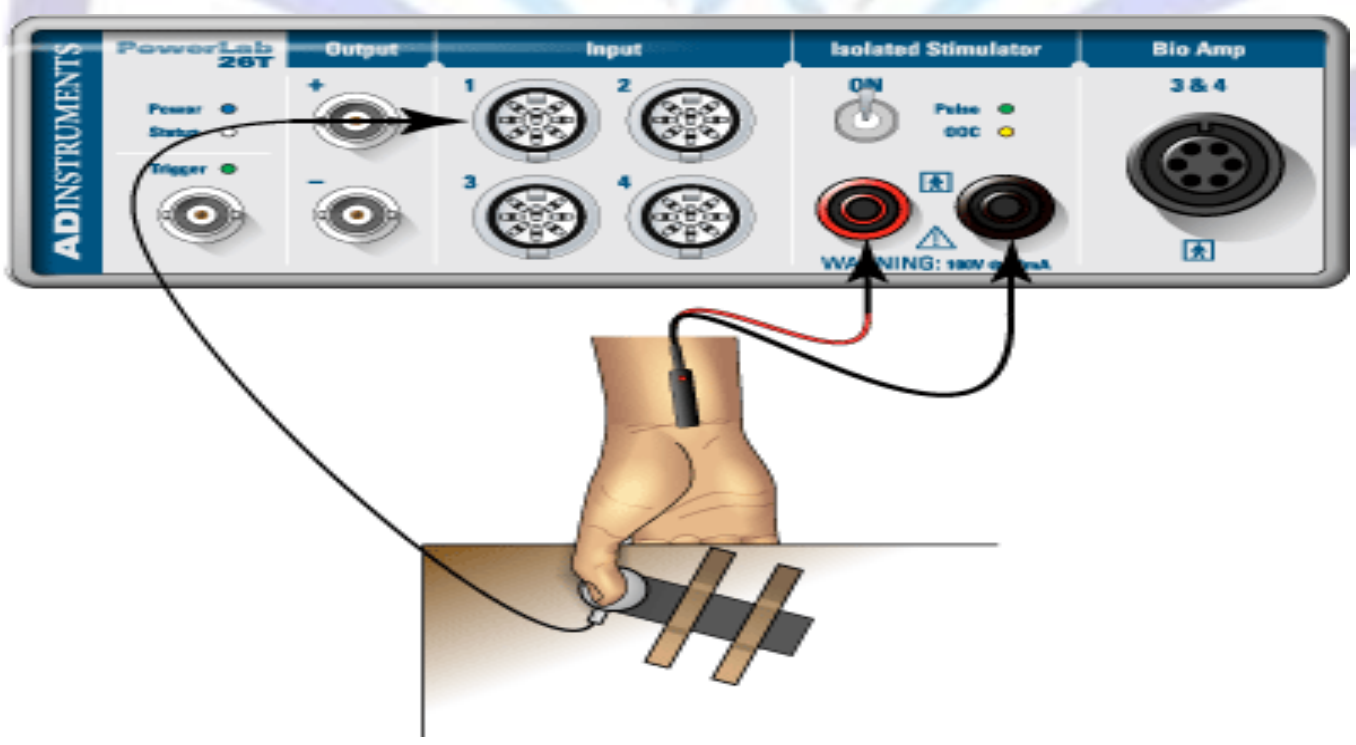




\section{Exercise 1}

Explore the effects of electrical stimuli using the nerves of the forearm and a Stimulating Bar Electrode.

\section{Procedure}

1. Check that the stimulator switch is off.

2. Place the Stimulating Bar Electrode over the volunteer's ulnar nerve at the wrist. The Stimulating Bar Electrode should be held in place along the axis of the arm with the red dot closest to the elbow.

3. In the Stimulator panel set current to $5 \mathrm{~mA}$.

4. Click Start then set the stimulator switch to $\mathbf{O N}$. The stimulator status light should now flash green, indicating that the chosen stimulus current is being passed through the subject's skin. If the light flashes yellow, current is not flowing properly.

5. Note the twitch contractions affecting the thumb and fingers. Examine the effect of small adjustments in the position of the electrodes, and locate the position giving the largest twitches. If no twitch occurs, increase the stimulus current.

6. Explore the motor and sensory results of stimulating at other places in the forearm. Each time you move the electrode to another location, wipe away the residual electrode cream from the skin to prevent short-circuiting. (Stimulation will be ineffective if the current flows along a surface layer of electrode cream rather than through the arm.)

You will probably find that effective stimulation will only occur when the two pads of the bar electrode are aligned along the arm's length. If the stimulus status light changes in color from green to yellow, you will need to put more electrode cream on the pads.

8. Try stimulating the ulnar nerve at the level of the elbow. The nerve passes behind a bony prominence (the medial epicondyle) on the humerus. At this location, the nerve is exposed to minor mechanical injury and is known to children as the "funny bone". Stimulation at this site gives large and obvious motor effects.

9. Click Stop and turn the stimulator switch off.

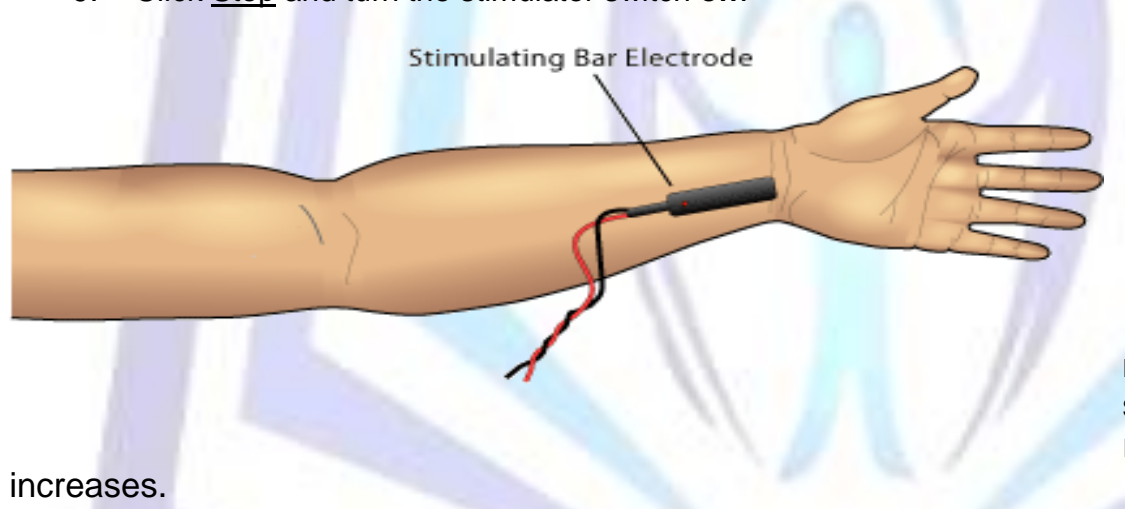

\section{Exercise 2}

Record and measure the muscular twitch response to nerve stimulation, and investigate recruitment as the stimulus strength

\section{Procedure}

\section{Finding the threshold}

1. Have the volunteer place his or her hand as shown here, with the fingers under the edge of the table and the edge of the thumb resting lightly on the pulse transducer. (If the table edge is too thick for the subject's hand, a plank or shelf may have to be used.)

2. Wipe the electrode cream from the subject's wrist.

3. Apply a small amount of electrode cream to the pads of the stimulating bar electrode.

4. Make the subject hold the electrode with the free hand firmly in place at the site for stimulation of the ulnar nerve at the wrist. Ensure that the edge of the subject's thumb is resting lightly on the transducer.

5. Ensure that the stimulus current is set to $1 \mathrm{~mA}$.

6. Set the stimulator switch to $\mathbf{O N}$.

7. Click Start.

LabTutor will stimulate, record for a fixed duration of 0.5 seconds and then stop automatically.

8. Increase the stimulus current to $2.0 \mathrm{~mA}$. 
9. Click Start.

10. Continue to increase the stimulus current in $1 \mathrm{~mA}$ steps, clicking Start each time.

For most subjects, the threshold stimulus at which a response is first observed is in the range 3-8 mA.

11. When you first see a response, add a comment to the recording, noting the subject's name and the stimulus current used.

\section{Recruitment}

12. Reduce the amplitude by $1 \mathrm{~mA}$.

13. Click Start.

14. Increase the amplitude in $0.5 \mathrm{~mA}$ steps, clicking Start each time and adding a comment, noting the current used.

15. Continue this until the response no longer increases.

For most subjects, this maximal stimulus is in the range 6-15 $\mathrm{mA}$.

16. Turn the stimulator switch off

\section{Analysis}

1. Locate the beginning of the recruitment recordings. Type into the first column of the table the current delivered to produce each response.

2. Move the cursor over the waveform and click on the peak of each response to transfer its value to the $\underline{\text { Value }}$ panel.

3. Drag the value to the appropriate cell in the table.

As you enter the data, the Graph panel will graphically display the relationship between stimulus current and response size.

Note the stimulus current at which the response no longer increases. This is called the 'maximal stimulus'.

\section{Exercise 2: Twitch response and recruitment}

\section{Study Questions}

1. Did you get a measurable twitch with a stimulus of $0 \mathrm{~mA}$ ? What does this tell you about the number of muscle fibers contracting at this stimulus current?

2. What was the smallest current required to produce a contraction (the threshold current)? What proportion of the fibers in the muscle do you think were contracting to produce this small response?

3. What was the smallest current required to produce the maximum (largest) contraction? What proportion of the fibers in the muscle do you think were contracting to produce this maximal response?

4. What do you conclude happened to the number of fibers contracting as the current was raised from threshold to that required to produce a maximal contraction?

5. Why does varying the stimulus strength affect the twitch force?

\section{Exercise 3}

Now stimulate with paired pulses, and investigate how varying the interval between the pulses affects the response. Use the same setup as in Exercise 2.

\section{Procedure}

1. Turn the Stimulator switch $\mathrm{ON}$

2. In the Stimulator panel, set the current to $5 \mathrm{~mA}$ greater than the maximal stimulus you determined in Exercise 2 .

Be sure to use the proper value for the present subject!

3. Check that the stimulus interval is set to $1000 \mathrm{~ms}$.

4. Click Start.

LabTutor will automatically deliver the stimulus and stop recording after a fixed duration of 3 seconds.

5. In the Stimulator panel decrease the stimulus interval to $500 \mathrm{~ms}$, and click Start.

6. Repeat this for intervals $200 \mathrm{~ms}, 150 \mathrm{~ms}, 100 \mathrm{~ms}$ and $50 \mathrm{~ms}$, noting the values in comments as you did above. When you have finished, turn the Stimulator switch OFF, ready for the next exercise. 


\section{Analysis}

1. Place the Marker on the baseline and the Waveform Cursor on the Peak to determine the peak amplitude of the first response.

2. Click on the peak to enter the force value into the Value panel.

3. Drag the value into the appropriate cell in the table.

4. Repeat steps 1-3 above for each response, at each stimulus interval.

\section{Exercises 3 \& 4: Summation and tetanus}

\section{Study questions}

1. The stimulus interval has a profound effect on the contraction force of the muscle. In your own words explain what happened when the muscle was stimulated in rapid succession. What was the minimum time required for the subject's muscle twitches to add together (summation)?

2. You use a short period electrical stimuli to observe muscle fibers contracting continuously (tetanus). Chemical agents can cause tetanus by interfering with the motor neurons. These agents include a toxin produced by the soil bacterium Clostridium tetani. One of the symptoms of these agents is called 'spastic paralysis'! Explain in your own words why these agents would be a bad thing for you and your muscles.

\section{Exercise 4}

Examine the effect of rapid stimulation and observe a short tetanic contraction.

\section{Procedure}

1. Ensure that the volunteer's hand and stimulus electrodes are placed as before, and turn the stimulator switch ON.

2. Check that the stimulus interval is set to $50 \mathrm{~ms}$. Set the number of pulses to 1.

3. Click Start.

LabTutor will automatically deliver the stimulus and stop recording after a fixed duration of 3 seconds.

4. Change the number of pulses to 2 and stimulate again.

5. Change the number of pulses to 3 and stimulate again.

6. If there was not too much discomfort, increase the number of pulses to 4 or 5 depending on the comfort level of the subject.

7. Turn the stimulator switch OFF and disconnect the stimulating bar electrode and the finger pulse transducer from the PowerLab.

\section{Analysis}

1. Place the Marker on the baseline and the Waveform Cursor on the Peak to determine the peak amplitude.

2. Click on the peak to enter the force value into the Value panel.

3. Drag the value into the appropriate cell in the table.

4. Repeat steps 1-3 above for each response

\section{Grip Force Calibration}

In the next exercise we investigate the decline in maximal force during a sustained contraction. Be sure the same volunteer performs both the calibration and Exercise 5 to achieve accurate results.

Make sure that you have removed the finger pulse transducer and the electrodes from the PowerLab.

1. Connect the plug of the grip force transducer to the Input 1.

2. The volunteer should loosely grip the hand dynamometer in the fist, as shown here.

3. Click Start.

4. The volunteer should squeeze the dynamometer as hard as possible for a second or two, and then relax.

5. Click Stop

Now, to calibrate for the strength of the volunteer: 
6. Click the trace at a time when the force is effectively zero, then click the Point 1 button in the Calibration panel. This will set the $0 \%$ grip force.

7. Click the trace at peak force, and click the Point 2 button in the Calibration panel. This represents $100 \%$ of the subject's grip force.

8. Click Apply.

9. Click Autoscale.

\section{Exercises 3 \& 4: Summation and tetanus}

\section{Study questions}

3. The stimulus interval has a profound effect on the contraction force of the muscle. In your own words explain what happened when the muscle was stimulated in rapid succession. What was the minimum time required for the subject's muscle twitches to add together (summation)?

4. You use a short period electrical stimuli to observe muscle fibers contracting continuously (tetanus). Chemical agents can cause tetanus by interfering with the motor neurons. These agents include a toxin produced by the soil bacterium Clostridium tetani. One of the symptoms of these agents is called 'spastic paralysis'! Explain in your own words why these agents would be a bad thing for you and your muscles.

\section{Exercise 5}

Measure the decline in maximal force during a sustained contraction, and examine some properties of muscular fatigue The grip force transducer should already be calibrated for the volunteer, as described in the previous page.

\section{Procedure}

1. Allow the volunteer to view the computer screen.

2. Click Start.

3. Ask the volunteer to maintain $25 \%$ maximal grip strength while watching the recorded trace.

4. After 20 seconds, tell the volunteer to relax.

5. Click Stop.

6. Wait for 30 seconds to allow recovery of muscle function.

7. Repeat steps $2-6$ for contractions of $50 \%, 75 \%$ and $100 \%$ of maximal grip strength.

8. Allow the volunteer to rest for two minutes.

9. Turn the volunteer away so that he or she cannot see the computer screen.

10. Click Start.

11. Ask the volunteer to produce a sustained maximal contraction.

12. After 8 to 10 seconds, or when the force has obviously declined, instruct them to try harder.

13. After a further 8 to 10 seconds, repeat the encouragement.

14. A few seconds later, ask the volunteer to relax.

15. Click Stop.

16. Click Start.

17. Ask the volunteer to produce a sustained maximal contraction. Every 8 to 10 seconds, allow the volunteer to relax very briefly (half a second), and then return to maximal contraction.

18. After 30 to 40 seconds click Stop.

19. Allow the volunteer to use his or her other hand if gripping the transducer has become painful. Turn the volunteer so that they can see the computer screen.

20. Click Start.

21. Ask the volunteer to produce a $50 \%$ contraction while watching the trace.

22. After 10 seconds, press the Enter key to enter a comment (to mark the time).

23. Have the volunteer close his or her eyes, and attempt to maintain exactly the same contraction force for the next 30 seconds. 
24. After the elapsed time, the volunteer should open their eyes, and adjust the contraction force back to $50 \%$.

25. Click Stop.

26. Examine the trace.

\section{Exercise 5: Muscle fatigue}

\section{Study Questions}

Fatigue is not well understood. Some factors that have been proposed to explain the fall in force during fatigue include: changes in the 'sense of effort', loss of 'central drive', failure of neuromuscular propagation, reduction in calcium release in excitation-contraction coupling, metabolic changes in the muscle, and reduction in muscle blood flow owing to compression of blood vessels.

1. Do your experiments help to decide which factors are important?

2. Almost all subjects will show a declining force (pseudo-fatigue), while their eyes are shut, that is very similar to fatigue. This is, however, not true fatigue, because the full $50 \%$ force can be exerted easily, as can be seen when the subject's eyes are opened again. What explanations can you think of for pseudo-fatigue?

\section{Experiment 9}

\section{Reflexes and Reaction Times}

\section{Introduction}

In this laboratory, you will investigate your reflexes and reaction times in response to a variety of stimuli and under a variety of conditions. You will examine some simple and complex reflexes from a volunteer, and look at reaction times from a volunteer given harmless visual and sound cues. You will also study the time required for a planned voluntary response to a cue.

\section{Learning Objectives}

By the end of today's laboratory you will be able to :

- $\quad$ Elicit a knee jerk

- Elicit pupillary reflexes

- Elicit an example of a flexion withdrawal reflex

- Discuss factors that affect reaction times
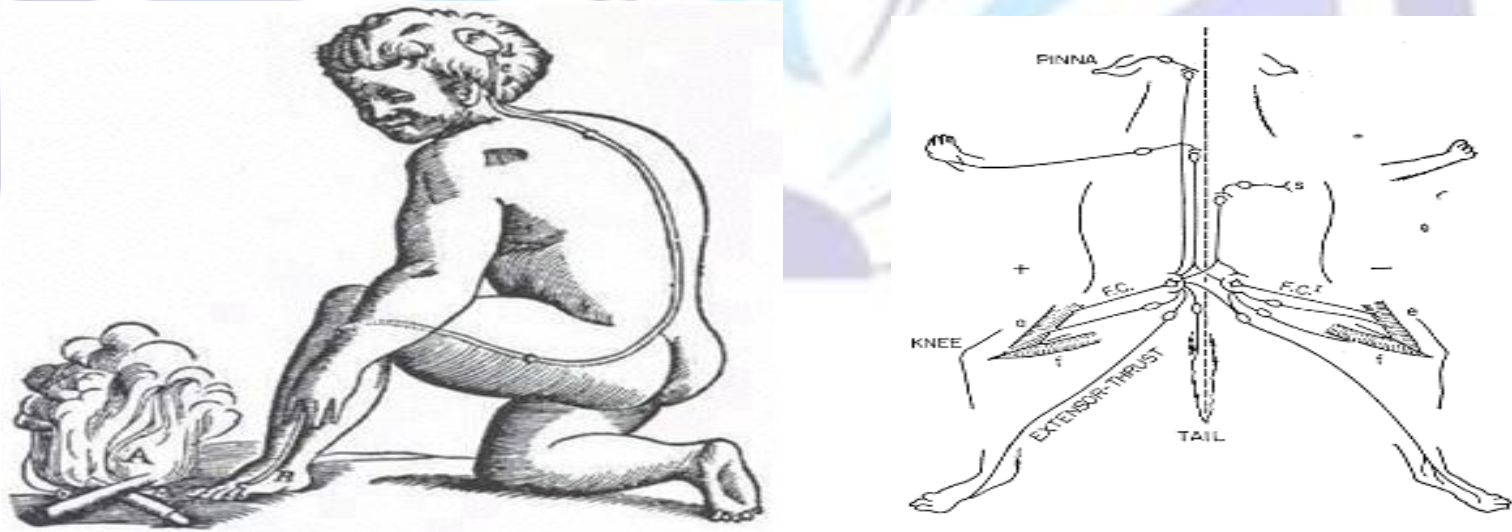

Figure 7 of De Homine (1662), by René Descartes (1594$1660)$, illustrates his concept of what is involved in a reflex response. The heat of the fire causes movements of animal spirits in hollow nerves. These open pores in the brain that result in spirits inflating the leg muscles so that the leg is removed from the source of the heat.
A diagram, from Sherrington's famous book, The Integrative Action of the Nervous System (first published in 1906), showing components of reflex behaviour. 
Setup

\section{If you do not have a tendon hammer and goniometer go straight to Exercise 1.}

\section{If a goniometer and tendon hammer are available:}

1. Attach the tendon hammer plug to Input 1 on the PowerLab.

2. Connect the plug from the Goniometer (joint angle sensor) to Input 2 on the PowerLab.

3. Attach the goniometer to the volunteer's leg so that it is centered on the knee joint. Adjust the Velcro straps so that they are comfortably snug.

\section{Exercise 1}

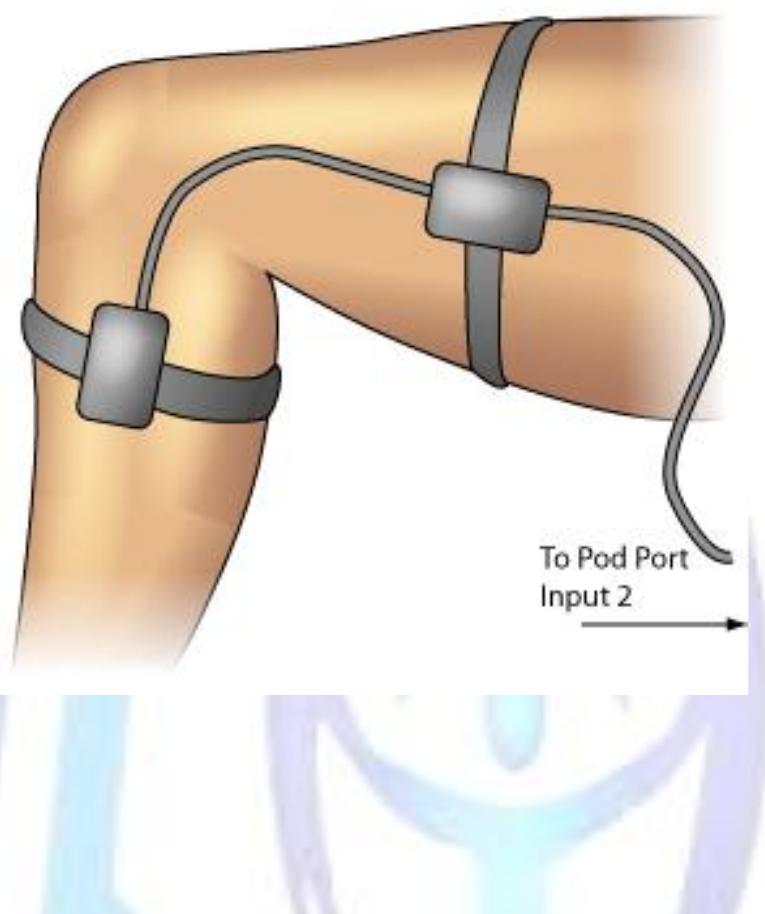

In this exercise, you will observe a myotatic reflex by stimulating the patellar tendon.

\section{Procedure}

If your lab is not equipped with a goniometer and tendon hammer, follow the alternative instructions for this exercise. Labtutor will automatically start recording for a fixed duration when the tendon hammer is tapped.

1. Ask the volunteer to sit in a chair and cross the leg that has the goniometer attached over the other leg, allowing the foot to swing backwards and forwards freely.

2. With the Tendon Hammer, tap the patellar tendon just below the knee to elicit the knee jerk response. Practice this exercise a few times so that you are comfortable in getting a reliable response.

3. Click Start. Using the Tendon Hammer, tap the patellar tendon of the volunteer.

4. Repeat this procedure four times, so that you have five blocks of data.

5. Now ask the volunteer to perform the 'Jendrassik maneuver'. To do this, cup and link the fingers of both hands, and then pull strongly outwards across their chest.

6. While the volunteer performs this maneuver, use the Tendon Hammer to tap the patellar tendon. Add the comment: 'Jendrassik' to each new block and record a total of five responses.

7. Click Stop.

\section{Analysis}

1. You will analyze and compare the latency and change in leg angle under normal conditions and while performing the Jendrassik maneuver.

2. Note that LabTutor is set up so that the stimulus is shown at time 0 in each block of data.

3. Scroll to the first block, performed under normal conditions. 
4. Measure the time between the stimulus and the start of muscle contraction (the latency) by moving the Waveform Cursor to the point where the goniometer trace $\left(\right.$ Angle $\left.^{\circ}\right)$ starts to deflect.

5. Click to place the time in the Time Value panel.

6. Drag the number from the Value panel into the appropriate Latency column of the Table.

7. Measure the amplitude of the knee jerk response for the same data by placing the Marker on the baseline data in the Angle channel and the the waveform cursor on the peak of the waveform.

8. Click to place the $\Delta$ Angle in the Angle Value panel.

9. Drag the number from the Value panel into the appropriate Angle column of the Table.

10. You must return the Marker to its dock before proceeding to the next block. Do this by clicking in its dock area.

11. Repeat this procedure for each of the data blocks

\section{Exercise 1: The myotatic reflex}

\section{Study Questions}

1. What effect does the Jendrassik maneuver have on the myotatic reflex?

2. What can you say about "simple" reflexes, given your results from the Jendrassik maneuver?

\section{Exercise 2}

The retina of the eye is able to respond to differences in light intensity over a very wide range. In bright light, the eye's sensitivity is low, but in dark conditions the sensitivity increases. Most of this adaptation occurs in the photoreceptor cells of the retina, but part of it results from regulation of the amount of light entering the eye through the pupil.

\section{Procedure}

1. Shade the volunteer's eyes for about 15 seconds.

2. Shine a light into one eye and note the response.

What is the response of the pupil when light is shone on it?

3. Repeat steps 1 and 2, but note the response of the pupil in the unstimulated eye. What was the response of the pupil in the opposite eye?

4. With normal lighting, ask the subject to look into the distance and then at an object held close up (about $10 \mathrm{~cm}$ from the eye).

What happens to pupil diameter when the eye is focused for near vision?

\section{Exercise 2: The pupillary light reflex}

\section{Study Questions}

1. What is the response of the pupil when light is shined on it?

2. What was the response of the pupil in the opposite eye?

3. What happens to pupil diameter when the eye is focused for near vision?

4. What is the apparent biological advantage of the pupillary light reflex?

\section{Exercise 3}

The flexion withdrawal reflex is not readily studied in human volunteers, because an unpleasantly painful stimulus is needed to evoke it. However, a little-known reflex involving an obscure muscle in the hand, the palmaris brevis muscle, exists that shares some features of the flexion reflex and is easily evoked. Everyone in the group should try this exercise.

\section{Procedure}

1. Cup your hand. Note a dimpling of the skin along the ulnar border (figure). This dimpling is the action of the palmaris brevis muscle.

In some people, it is difficult to see the dimpling of the skin; reflex effects will then be hard to demonstrate.

2. With the palm of your hand facing up and the hand relaxed, press with your fingernail over the pisiform bone (figure). Firm pressure causes a reflex contraction of the palmaris brevis muscle.

3. Try to find other bony prominences in the hand at which the reflex can be elicited. 
4. Attempt to contract the palmaris brevis muscle voluntarily, without moving your little finger. Most people find this difficult or impossible. However, with extensive practice the movement can be learned.

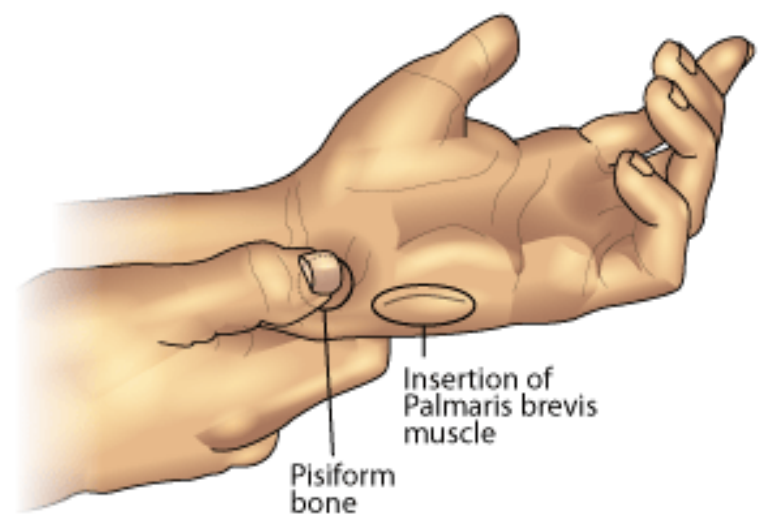

Palmar view of the left hand, showing where the palmaris brevis muscle dimples the skin and the location of the pisiform bone.

\section{Setup}

In this part of the laboratory, you will measure a volunteer's reaction time to a visual cue.

1. Disconnect the Electronic Tendon Hammer and Goniometer from the PowerLab.

2. Connect the push-button switch to the socket for Input 1.

3. Connect the finger pulse transducer to the socket for Input 2.

4. Wrap the Velcro strap around the finger pulse transducer to cover its diaphragm. This protects the transducer against hard tapping.

5. Place the pulse transducer on the laboratory bench with the diaphragm facing up, in a place where it will not be bumped accidentally. Keep the transducer in place with a piece of adhesive tape across the cable.

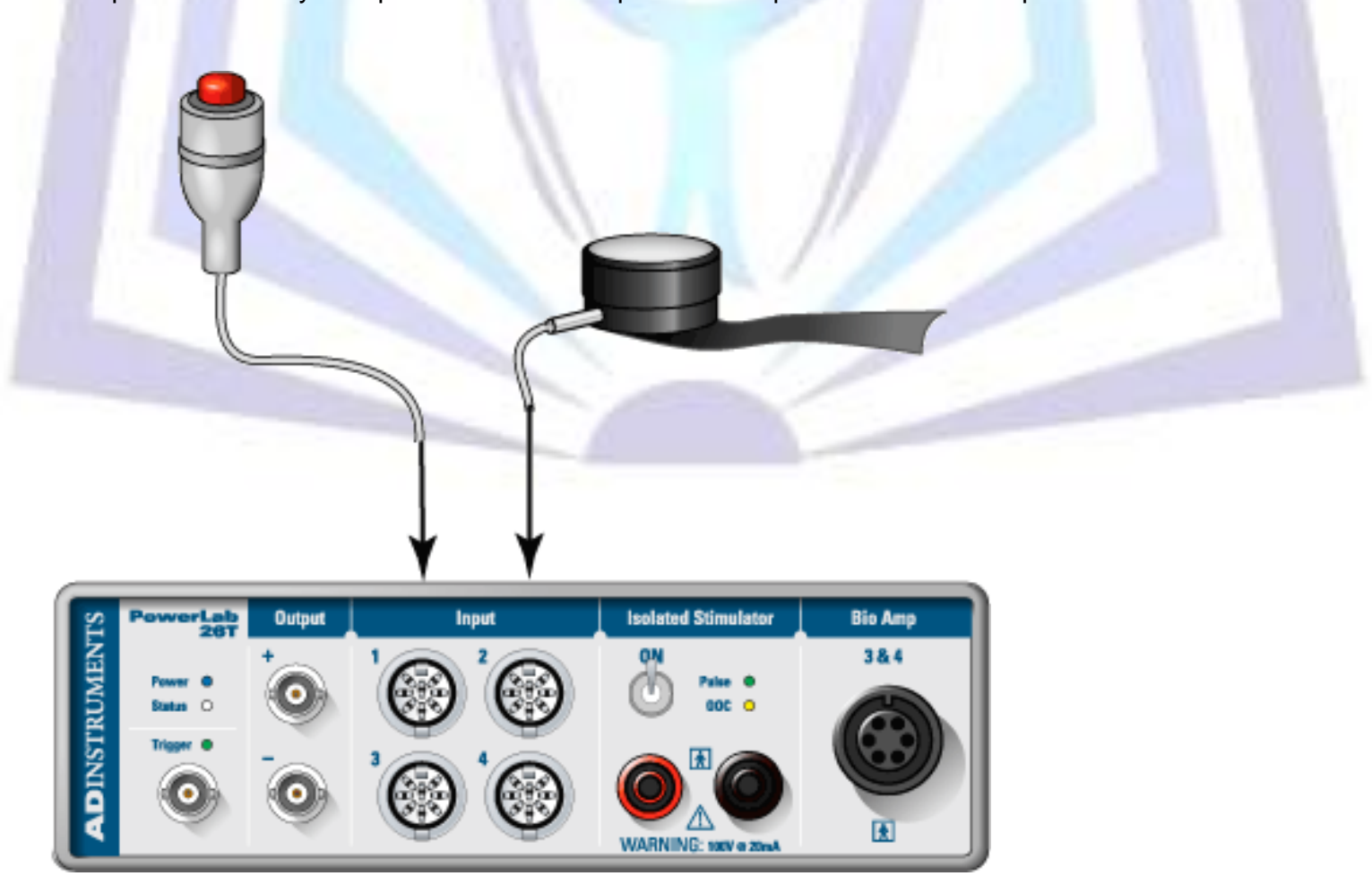

\section{Exercise 4}

In this exercise, you will evaluate the reaction time of a member of your group. 


\section{Procedure}

1. Instruct the subject to click the push-button switch as soon as you are seen to tap the transducer.

2. Hold one hand just over the transducer, but not touching it.

3. Click Start.

The system will record for 0.75 seconds when it receives a signal from the finger pulse transducer.

4. After a delay of 1 to 4 seconds, tap the transducer without warning the subject.

5. Repeat steps $3-4$ a total of ten times, ensuring that the taps are given without warning, at unpredictable intervals in the range of 1 to 4 seconds.

6. Recording will stop automatically after ten records have been recorded.

\section{Analysis}

1. Move the Waveform Cursor until it is over the start of the response. [The reaction time is the time from the stimulus (time zero) to the start of the response.]

2. Click to place the time in the Value panel.

3. Drag the number from the Value panel into the 'Random' column of the table.

4. Repeat the measurement for all ten trials.

5. Delete the longest and shortest values, the table will automatically update and average the remaining eight values to give the mean reaction time.

\section{Exercise 5}

In this part of the experiment, you will measure the reaction time to a visual cue given immediately after a verbal prompt.

\section{Procedure:}

1. Click Start. The system will begin recording when it receives a signal from the transducer.

2. After a delay of 1 to 4 seconds, warn the volunteer verbally by saying "ready", immediately before tapping the transducer.

3. Repeat steps 1 - 2 a total of ten times, ensuring that the taps are given with a warning, but at unpredictable intervals in the range of 1 to 4 seconds.

4. Recording stops automatically after ten records have been recorded.

\section{Analysis}

1. Move the Waveform Cursor until it is over the start of the response. [The reaction time is the time from the stimulus (time zero) to the start of the response.]

2. Click to place the time in the Value panel.

3. Drag the number from the Value panel into the 'With Warning' column of the table.

4. Repeat the measurement for all ten trials.

5. Delete the longest and shortest values, the table will automatically update and average the remaining eight values to give the mean reaction time.

\section{Exercise 6}

In this part of the laboratory, you will measure the subject's reaction time to a cue given at regular intervals.

\section{Procedure}

1. Click Start. The system will begin recording when it receives a signal from the transducer.

2. Instead of a verbal warning, tap the transducer in a regular rhythm, such as every 2 seconds.

3. Repeat steps 1 and 2 a total of ten times.

4. Recording stops automatically after ten records have been recorded. 


\section{Analysis}

1. Move the Waveform Cursor until it is over the start of the response. [The reaction time is the time from the stimulus (time zero) to the start of the response.]

2. Click to place the time in the Value panel.

3. Drag the number from the Value panel into the 'Regular' column of the table.

4. Repeat the measurement for all ten trials.

5. Delete the longest and shortest values, the table will automatically update and average the remaining eight values to give the mean reaction time.

\section{Exercise 7}

In this exercise, you will measure the subject's reaction time while doing mental arithmetic.

\section{Procedure}

1. Click $\underline{\text { Start. }}$ The system will begin recording when it receives a signal from the transducer.

2. Ask the subject to count down from 100 by 7 , i.e., starting from 100 and subtracting 7 repeatedly as fast as possible. The subject should say each number aloud $(100,93,86)$.

3. After a delay of 1 to 4 seconds and without warning, tap the transducer.

4. Again, the subject should click the push-button switch as soon as you are seen to tap the transducer.

5. Repeat steps 2 - 4 a total of ten times.

6. Recording stops automatically after ten records have been recorded.

\section{Analysis}

1. Move the Waveform Cursor until it is over the start of the response. [The reaction time is the time from the stimulus (time zero) to the start of the response.]

2. Click to place the time in the Value panel.

3. Drag the number from the Value panel into the 'With Distraction' column of the table.

4. Repeat the measurement for all ten trials.

5. Delete the longest and shortest values, the table will automatically update and average the remaining eight values to give the mean reaction time.

\section{Exercise 8}

In this exercise, you will measure the subject's reaction time to sound.

\section{Procedure}

1. Have the subject face away from both the finger pulse transducer and the computer screen to avoid visual cues but be close enough to hear a vigorous tap on the transducer.

2. Experiment with different ways of tapping, to find one which gives a sound that can be clearly heard by the subject, but without risk of damaging the transducer. Try tapping the bench and the transducer simultaneously. Alternatively, cover the transducer with a piece of folded paper, and flick it sharply.

3. Click Start. The system will begin recording when it receives a signal from the transducer.

4. After a delay of 1 to 4 seconds, tap the transducer without warning the volunteer.

5. Again, the subject should click the push-button switch as soon as the tap on the transducer is heard.

6. Repeat steps 3 - 4 a total of ten times.

7. Recording stops automatically after ten records have been recorded.

\section{Analysis}

1. Move the Waveform Cursor until it is over the start of the response. [The reaction time is the time from the stimulus (time zero) to the start of the response.]

2. Click to place the time in the Value panel.

3. Drag the number from the Value panel into the 'Auditory Cue' column of the table. 
4. Repeat the measurement for all ten trials.

5. Delete the longest and shortest values, the table will automatically update and average the remaining eight values to give the mean reaction time.

\section{Exercise 8: Reaction time to auditory cues}

\section{Study Questions}

1. Based on your data, is the mean reaction time the same under all conditions?

2. Which conditions increase the reaction time, and which decrease it?

3. Do you think that the difference in reaction time between auditory and visual cues is really due to different processing times in the brain?

4. Could the difference in reaction times just be due to inherent variability in the experimental method?

\section{Experiment 10}

\section{Cardiovascular Effects of Exercise}

\section{Introduction}

In this laboratory, you will record the electrocardiogram (ECG) and the finger pulse from a healthy volunteer, and compare the recordings made when the volunteer is at rest and immediately after exercise. This experiment is suitable for undergraduates with some knowledge of the PowerLab system, and previous experience with the recording of an ECG.

\section{Learning Objectives}

By the end of today's laboratory you will be able to:

- Describe how heart rate and finger pulse change after moderately vigorous, short-term exercise

- Describe how heart rate and finger pulse change after finger exercise

- List factors that control heart rate before, during and after exercise

- List factors that control blood flow to tissues before, during and after exercise

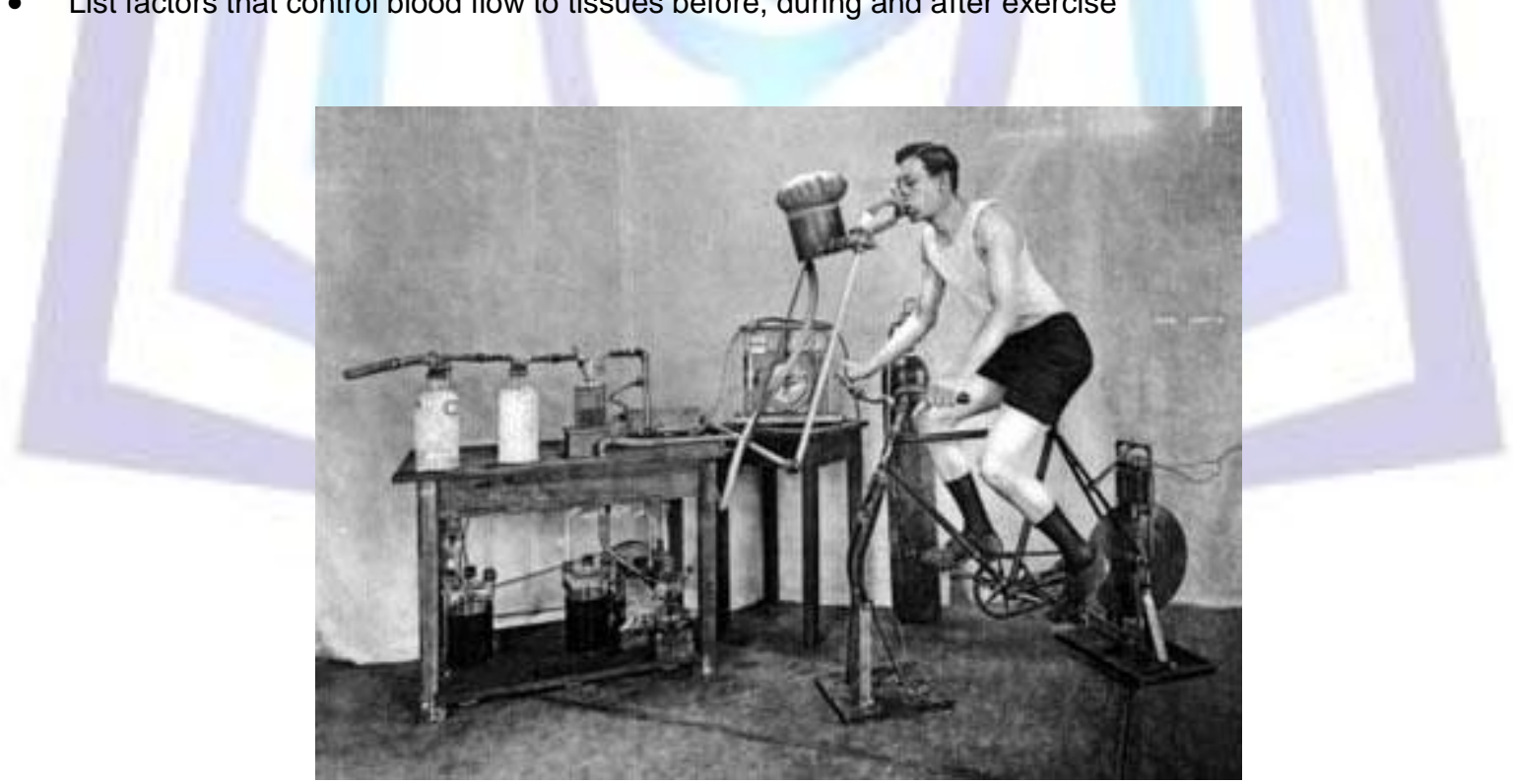

Original bicycle ergometer used in Benedict's experiments to assess exercise energy metabolism in the early 1900 s

\section{Procedure}

1. Make sure the PowerLab is connected and turned on.

2. Attach the Finger Pulse Transducer to a middle finger.

3. Connect the Finger Pulse Transducer to Input 1.

4. Remove any watches and/or jewelry from your wrists and ankles. 
5. Connect the electrode lead wires to Earth, and the $\mathrm{CH} 1 \mathrm{NEG}$, and POS on the Bio Amp cable.

6. Plug the Bio Amp cable into the Bio Amp input.

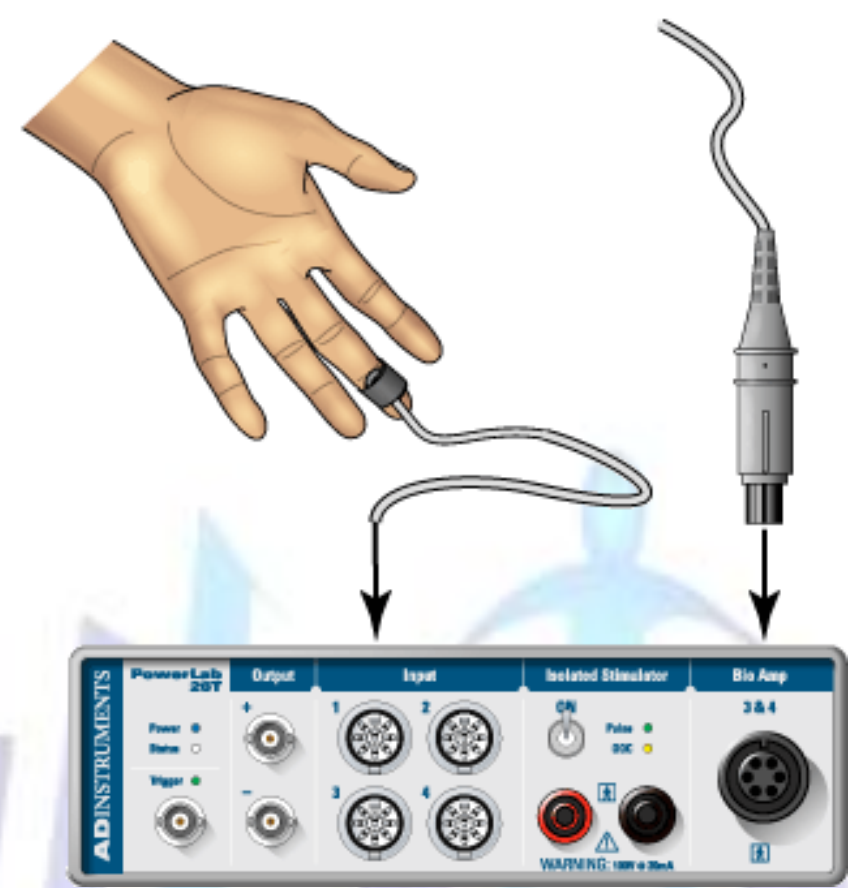

\section{Standard Connection}

Attach the positive electrode to the left wrist, the negative to the right wrist, and the ground to the right leg.

1. Using a pen, mark each point where electrodes will be placed.

2. Clean the skin with alcohol swabs and lightly abrade the area with abrasive gel or a pad. This reduces the electrical resistance of the outer layer of skin and ensures good electrical contact.

3. If you are using the Reusable Clamp Electrodes, apply a small amount of electrode cream to the electrodes before attaching. Electrode cream is not necessary if you are using disposable electrodes which have electrode gel on them already.

4. If, after looking at the signal during the first exercise, you find that this does not produce a good signal, try the alternative method.

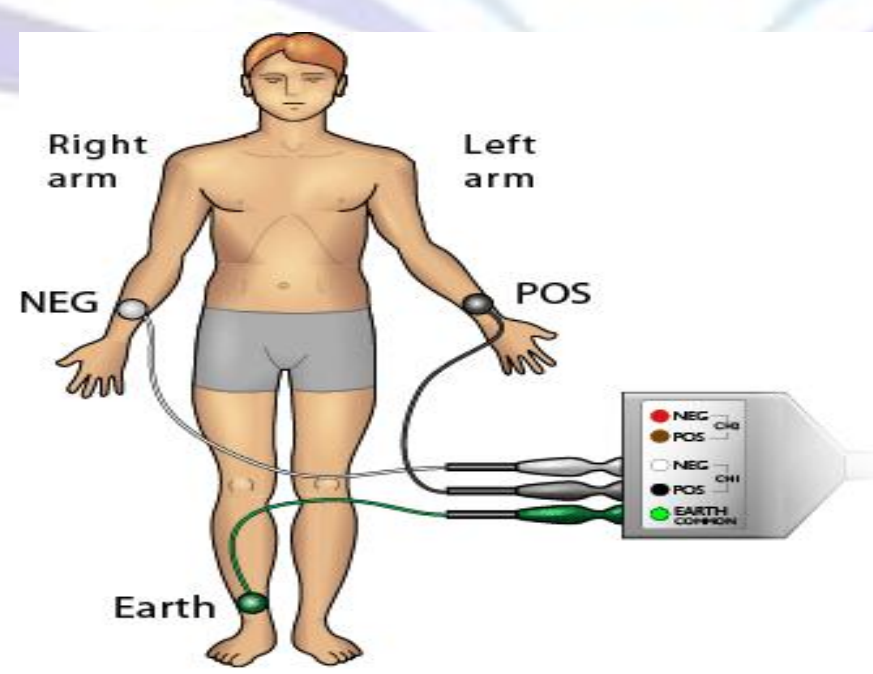

\section{Preliminary Exercise}

You will record an ECG and identify artifacts in the signal resulting from movement. 


\section{Procedure}

1. The subject should relax and sit as still as possible to minimize signal artifacts due to movements.

2. Click Start and record for a few seconds.

3. Click Stop.

If the ECG cannot be seen, check that all three electrodes are correctly attached. If the signal is noisy and indistinct, make sure that the subject is relaxed; consider using the alternative attachment positions.

4. Repeat step 2. Once you have a satisfactory ECG, proceed to the next exercise.

\section{Exercise 1}

Now that you have established a good clear signal from the preliminary exercise, you will record the ECG and pulse from a resting subject.

\section{Procedure}

1. Type the subject's name into the Comment panel with 'Resting ECG'.

2. Click Start, add the comment and record for about 30 seconds.

3. Click Stop.

4. Disconnect the finger pulse transducer and the Bio Amp cable from the PowerLab. The subject should gather them, and the ECG leads up and hold them.

5. Now the subject should exercise for at least two minutes: for example, two minutes of step-up exercise, or running up four flights of stairs.

Remember that the ECG leads are still attached to the electrodes, so exercise carefully (so as not to break the leads or move the electrodes), but vigorously enough to elevate your heart rate.

6. While the subject is exercising, enter 'Recovery' into the Comment panel.

7. Immediately after exercise, the subject should sit down and relax.

8. Reconnect the Bio Amp cable to the PowerLab, and the finger pulse transducer to Input 1.

9. Click Start.

10. Add the comment, and record for two minutes or until the heart and breathing rates have returned to normal.

11. Click Stop.

\section{Analysis}

1. Your ECG trace should resemble the one shown here.

LabTutor is set up so that channel 3 shows the heart rate calculated from the RR interval of the ECG. Channel 4 shows the calculated pulse amplitude.

2. Move the Waveform Cursor to a representative cycle before exercise.

3. Click to place the Heart rate and Pulse amplitude in the Value panels.

4. Drag the number from the 'Heart rate' Value panel into the appropriate column of the table.

5. Next drag the number from the 'Pulse amplitude' Value panel into the appropriate column of the table.

6. Repeat steps 3 to 5 for each of the times after exercise that are shown in the table.

\section{Analysis (continued)}

1. Now you will measure the intervals that make up a cardiac cycle under resting conditions and after exercise.

2. Scroll to the resting ECG.

3. Expand the view of your ECG by setting the compression to $5: 1$.

4. To determine the PR interval, place the marker at the beginning of a P wave and move the Waveform Cursor to the start of the QRS complex. Click and use the Value panel to transfer the time to the appropriate cell in the table.

5. To determine the QRS interval, place the marker at the beginning of the QRS complex and move the Waveform Cursor to the end of the QRS complex. Click and use the Value panel to transfer the time to the appropriate cell in the table. 
6. To determine the ST interval, place the marker at the end of the QRS complex and move the Waveform Cursor to the end of the T wave. Click and use the Value panel to transfer the time to the appropriate cell in the table.

7. To determine the TP interval, place the marker at the end of the T wave and move the Waveform Cursor to the beginning of the next $\mathrm{P}$ wave. Click and use the Value panel to transfer the time to the appropriate cell in the table.

8. Repeat steps 4 to 7 for each of the post exercise columns.

\section{Exercise 1: ECG during rest and after exercise}

\section{Study Exercise}

Add comments to the recording to indicate the location of each of the following: the P-wave, the QRS complex, and the T-wave.

\section{Study Questions}

1. What happened to the R-R interval and the heart rate after exercise?

2. The R-R interval consists of the sum of QRS, S-T, T-P and P-R. Which of these became shorter when the heart rate increased?

3. Immediately after exercise, was the amplitude of the pulse smaller or larger than in the resting record?

4. What happened to the pulse amplitude during recovery from exercise?

5. During exercise, blood flow in the skin and finger pulp is usually reduced by sympathetic vasoconstrictor nerve activity. After exercise, however, skin and finger blood flow may be increased. Why is the blood flow to extremities reduced during exercise? Why does it increase to higher than normal levels during recovery? What other factors may influence the supply of blood to the skin and fingers during and after exercise?

6. Changes in the cardiovascular system are only some of a number of changes that occur in the body during and after exercise. What other physical changes did you observe in the volunteer?

\section{Exercise 2}

You will investgate changes in the pulse associated with hand exercise.

\section{Procedure}

1. Disconnect the ECG leads and attach the finger pulse transducer to the subject's middle finger.

The subject should remain still and relaxed.

2. Click Start and record for 20 seconds; during this time, enter the comment 'Resting.'

3. Click Stop.

4. The subject should now:

- grasp a rubber squeeze-ball in the palm of the hand, to which the finger pulse transducer is attached

- rhythmically squeeze it for a few minutes until the forearm muscles fatigue ○ stop exercising

5. Click Start.

6. Record for two minutes or until the amplitude of the finger pulse signal has attained a reasonably constant level for one minute; during this time, enter the comment 'Recovery.'

7. Click Stop.

\section{Analysis}

LabTutor is set up so that Channel 2 shows the pulse rate and Channel 3 shows the pulse amplitude.

1. First, use the Waveform Cursor to measure the pulse rate and amplitude in the absence of exercise.

2. Move the Waveform Cursor to a representative pulse rate. Then click to place the number in the Value panel.

3. Drag the number from the 'Pulse rate' Value panel into the appropriate column of the table.

4. Next drag the number from the 'Pulse amplitude' Value panel into the appropriate column of the table.

5. Repeat steps 2-4 for your post-exercise data at 10, 30, 60 and 120 seconds. 


\section{Exercise 2: Pulse after hand exercise}

\section{Study Questions}

1. Immediately after hand exercise, was the amplitude of the pulse smaller or larger than in the resting record?

2. What happened to the pulse amplitude during recovery from hand exercise?

3. The muscles active during hand exercise are mainly in the forearm, with a few small muscles in the hand. Would you expect blood flow to these muscles to increase during exercise?

4. There are no muscles in the distal segment of the fingers. Would you expect the increased muscle blood flow in exercise to affect the blood flow under the finger pulse transducer? 